\title{
55. MAJOR ELEMENT CHEMISTRY OF THE JAPAN TRENCH SEDIMENTS, LEGS 56 AND 57, DEEP SEA DRILLING PROJECT
}

\author{
Ryuichi Sugisaki, Department of Earth Sciences, Nagoya University, Nagoya, Japan
}

\begin{abstract}
The bulk chemical composition of 257 argillaceous sediments from cores around the Japan Trench which were drilled during Legs 56 and 57 was determined and examined. These samples are generally enriched in $\mathrm{SiO}_{2}$ and impoverished in $\mathrm{MnO}$, compared with typical pelagic sediments. $\mathrm{SiO}_{2}$ is negatively correlated with other oxides. Statistical treatment of data shows that pure silica varies inversely with $\mathrm{TiO}_{2}$ and $\mathrm{Al}_{2} \mathrm{O}_{3}$. The silica was derived mainly from planktonic siliceous organisms such as diatoms and radiolarians. Siliceous tests of these organisms disappear during progressive diagenesis, but the resolved silica redeposited in the sediments and the total silica content of the sediments remain constant. Higher $\mathrm{SiO}_{2}$ in some samples may be due to increased volcanic glass content. On the west slope of the trench, much ferric iron was deposited but not oxidized. In contrast to iron, manganese originally present in the sediments was apparently reduced and not precipitated in this hemipelagic region. Abundant land-derived organic materials maintain a weakly reducing environment within the sediment, which permits the precipitation of ferric iron but not of manganese. The mutual relationship of conservative elements such as $\mathrm{SiO}_{2}, \mathrm{TiO}_{2}$, and $\mathrm{Al}_{2} \mathrm{O}_{3}$ indicates that sediments of the west slope of the trench were derived mostly from the land area to the west and that the original materials were chemically homogenized and diluted by biogenic silica. In contrast, the chemistry of core from the trench outer slope (Site 436) shows a vertical trend: $\mathrm{SiO}_{2}$ progressively decreases downhole, whereas $\mathrm{MnO}$ and the $\mathrm{Fe}_{2} \mathrm{O}_{3} / \mathrm{FeO}$ ratio increase significantly. The deeper samples are very similar to the pelagic sediments from the Pacific Plate, whereas upper samples resemble the trench inner slope sediments. The vertical trends at this site are plausibly explained by the progressive movement of the ocean plate toward the Japan Trench. When the oceanic plate was in the Central Pacific Ocean, much $\mathrm{MnO}$ and $\mathrm{Fe}_{2} \mathrm{O}_{3}$ were deposited under an oxidizing environment with slow sedimentation, owing to the paucity of organic materials. As the ocean plate moved westward, nearer to the Japanese Islands, hemipelagic sediment derived from these islands were deposited over the pelagic sediments.

No pelagic sediments were detected geochemically in the deposits of the trench inner slope. If the offscraping of the sediment of the oceanic plate is the dominant process at the plate convergence, significant amounts of deep oceanic pelagic sediments that are being scraped off from the oceanic plate should be contained in the trench inner slope. Since the pelagic sediments are known to exist on oceanic crust coming into the trench, it may be that the pelagic sediments are subducted without offscraping against the landward wall of the trench. However, the entire sequence underlying the trench inner slope was not penetrated at any site.
\end{abstract}

\section{INTRODUCTION}

The Japan Trench-island arc system is a typical example of plate convergence accompanied by subduction of oceanic plate beneath the continental plate. The chemical feature of sediments in the trench can be one of the most important keys to an understanding of the subduction mechanism. The bulk chemical composition of the 257 samples drilled during Legs 56 and 57 was determined and examined in this context.

The chemical aspects of argillaceous sediments from piston cores around the Japanese Islands have already been published (Sugisaki, 1978, 1979; Sugisaki and Hon$\mathrm{za}$, in press; Sugisaki, in press a). Here I compare the data of Legs 56 and 57 with those from several regions around the Japanese Islands and attempt to place some 
chemical constraint on models for the observed sediment chemical variations in the DSDP sites.

\section{EXPERIMENTAL METHODS AND RESULTS}

The analytical procedure of marine argillaceous sediments involves much work, because the determination of carbonates, interstitial salts, organic materials, and total water content is needed for correction of the data. Furthermore, the usual method of $\mathrm{FeO}$ determination with oxidation-reduction titration is not applicable to the marine sediments, because the endpoint of the titration is very vague owing to the presence of organic materials. The improved method is described elsewhere in detail (Sugisaki, in press b) and it is outlined in the following.

The samples for this study were dried at $80^{\circ} \mathrm{C}$, ground, and subsequently split into fractions. One fraction was analyzed with an automatic X-ray fluorescence spectrometer JOEL-JSX-100S for $\mathrm{Si}, \mathrm{Ti}, \mathrm{Al}, \mathrm{Fe}, \mathrm{Mn}, \mathrm{Ca}$, $\mathrm{Mg}, \mathrm{Na}, \mathrm{K}$, and $\mathrm{P}$. The details of the method have been published (Sugisaki et al., 1977). Other fractions were analyzed for total water, ignition loss, dissolved chlorine, ferrous iron, and carbonate, according to the methods noted in Table 1. The distribution of the samples and the analytical results are given in Table 1. Table 2 lists the silicate composition of samples recalculated by excluding carbonate, salts, water, and residual materials (organic matter and others).

\section{GENERAL CHEMICAL CHARACTERISTICS OF THE SEDIMENTS}

The average chemical composition of the sediments and the standard deviation at each site are shown in Table 3 . Their similarity is striking. The small standard deviation from the average value reveals a particularly high degree of uniformity in the argillaceous sediments with respect to geochemical characteristics, in spite of the great length of the core.

For comparison, five sets of averages are listed in Table 3, namely that of pelagic sediments from the East Pacific Ocean and those of argillaceous sediments from four regions around the Japanese Islands. The DSDP samples, as well as others, are generally enriched in $\mathrm{SiO}_{2}$ and impoverished in $\mathrm{MnO}$, in comparison with typical pelagic sediments. Specifically the DSDP samples are higher in $\mathrm{SiO}_{2}$ than those from the other four regions around the Japanese Islands. Among the former, Site 436 is chemically different from the rest. It is located on the oceanic plate east of the Japan Trench, whereas Sites $434,435,438,439,440$, and 441 are on the landward side. In the next four sections, I discuss the chemical features of sediments from the landward side of the trench.

\section{ANOMALOUS MANGANESE CONTENT AT SITE 434}

The average $\mathrm{MnO}$ content at Site 434 is about twice that of the other sites (Table 3). Samples with more than 0.1 per cent $\mathrm{MnO}$ contain mostly $\mathrm{CaCO}_{3}$ (Figure 1). Some samples containing $\mathrm{CaCO}_{3}$ also show high total $\mathrm{Fe}$ content as $\mathrm{Fe}_{2} \mathrm{O}_{3}$. There are marlstones at several lo- cations in the core, and they may be responsible for the high $\mathrm{MnO}$ content at this site. Furthermore, Okada (this volume) described micritic calcareous nodules from this site. They are enriched in manganese and iron. Samples with high total iron and $\mathrm{MnO}$ content may indicate incipient formation of manganiferous calcareous nodules, although the $\mathrm{MnO}$ /total $\mathrm{Fe}$ ratios are considerably different from those of the calcareous nodules described by Okada.

\section{ORIGIN OF EXCESS SILICA}

Table 2 indicates the high $\mathrm{SiO}_{2}$ content in sediments at each site of the west slope of the trench, some of the samples containing 80 per cent $\mathrm{SiO}_{2}$. This value is high when compared with average pelagic sediments from the Pacific Ocean, with argillaceous sediments near the Japanese Islands (Table 3), and with typical shales. Plausible explanations for the chemical characteristics are (1) source materials inherently high in silica and (2) excess silica deposited along with the sediments.

In order to examine the possibilities, correlation coefficients between $\mathrm{SiO}_{2}$ and other oxides were calculated for each site (Table 4). $\mathrm{SiO}_{2}$ correlates negatively with other oxides. The coefficients for $\mathrm{Al}_{2} \mathrm{O}_{3}$ and $\mathrm{TiO}_{2}$ are conspicuous. The relation of $\mathrm{SiO}_{2}$ versus $\mathrm{Al}_{2} \mathrm{O}_{3}$ at Site 438 is shown in Figure 2. The regression line calculated by the least squares method is $\mathrm{SiO}_{2}=100.1-2.12 \mathrm{Al}_{2} \mathrm{O}_{3}$. This formula gives 100.1 per cent of silica when $\mathrm{Al}_{2} \mathrm{O}_{3}$ is absent. The regression lines calculated in the same way for each site give $\mathrm{SiO}_{2}$ values between 99 and 101 per cent, except for Site 436, where $\mathrm{Al}_{2} \mathrm{O}_{3}$ is absent (Table 5). Considering the analytical error, the range of $\mathrm{SiO}_{2}$ is surprisingly narrow. This indicates that pure silica from a separate source is diluting $\mathrm{Al}_{2} \mathrm{O}_{3}$ during sedimentation, because the igneous and metamorphic rocks as well as the typical sedimentary rocks regarded as source materials of the sediments do not have the same relation between $\mathrm{Al}_{2} \mathrm{O}_{3}$ and $\mathrm{SiO}_{2}$. The regression line at Site 436 is different from those at the other sites, which will be discussed later.

Percentages of $\mathrm{SiO}_{2}$ for Site 438 when other oxides are absent were calculated from the regression lines for each oxide versus $\mathrm{SiO}_{2}$ by the same method as for $\mathrm{Al}_{2} \mathrm{O}_{3}$ (Table 6). Each value of $\mathrm{SiO}_{2}$ for $\mathrm{TiO}_{2}, \mathrm{Al}_{2} \mathrm{O}_{3}, \mathrm{FeO}$, $\mathrm{MgO}$, and $\mathrm{K}_{2} \mathrm{O}$ approximates 100 per cent, whereas that for $\mathrm{Fe}_{2} \mathrm{O}_{3}, \mathrm{MnO}, \mathrm{CaO}$, and $\mathrm{P}_{2} \mathrm{O}_{5}$ is much lower than 100 per cent. This implies that the excess silica was simply added to the elements of the first group but that the elements in the second group coprecipitated with silica. The precipitation of $\mathrm{Fe}_{2} \mathrm{O}_{3}$ and $\mathrm{MnO}$ will be discussed later.

The origin of the excess silica may be explained in terms of (1) acidic glass ejected from active volcanoes, (2) precipitation of siliceous organisms, and (3) inorganic precipitation of silica from sea water. The description of the drilled cores (site chapters, this volume, Pt. 1) shows the presence of significant amounts of such siliceous organisms as diatoms, sponges and radiolarians. The percentages of these organisms were not determined in the samples for chemical analyses. The estimates of percentages of these organisms (smear slides; see site 
TABLE 1

Chemical Composition of Sediments

Sample

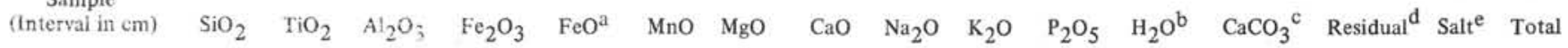

Hole 434

$1-4,80-82$

$2-1,100-104$

4-1, 80-84

$5-1,120-124$

6, CC, 31-35

$7-1,44-48$

$7-2,49-53$

$7-2,50-54$

$9-1,46-5$

9-2, 13-17

$12-1,80-84$

$15-1,75-80$

$15-2,82-86$

$16-1,22-26$

$17-1,20-22$

$19-1,36-40$

$19-2,56-60$

20-1, 96-99

$22-1,115-118$

$23-1,66-68$

24-1, 86-90

$25-1,54-58$

$26-1,40-44$

27-1, 86-90

$28-2,76-79$

$30-1,81-84$

$31-1,43-47$

$33-1,75-79$

$33-1,84-88$

$33-2,84-88$

Hole 434A

$1-1,56-60$

$1-5$

Hole 434B

$4-1,42-46$

$7-1,35-39$

$8-2,66-70$

9-2, 60-64

$10-1,80-84$

$11-2,76-80$

$15-2,100-104$

$16-1,90-94$

$17-1,76-80$
$19-1,110-114$

$19-1,110-$

20-1, 60-64

$25-1,56-60$

$26-1,100-104$

$30-1,82-84$

$32-1,84-88$

$33-1,86-90$

34-2, 60-64

$35-1,86-90$

$36-1,15-19$ $\begin{array}{ll}66.5 \mathrm{i} & 0.49 \\ 65.77 & 0.52\end{array}$

$\begin{array}{lll}65.77 & 0.52 & 11.99\end{array}$

$\begin{array}{lll}67.20 & 0.47 & 11.02\end{array}$

$\begin{array}{lll}64.58 & 0.57 & 13.18\end{array}$

$56.29 \quad 0.42$

$69.41 \quad 0.42$

$\begin{array}{ll}71.73 & 0.43 \\ 71.48 & 0.42\end{array}$

$\begin{array}{ll}61.12 & 0.61\end{array}$

$\begin{array}{ll}69.76 & 0.44\end{array}$

$\begin{array}{ll}74.17 & 0.39\end{array}$

$69.79 \quad 0.43$

$\begin{array}{ll}65.08 & 0.59\end{array}$

$\begin{array}{ll}64.46 & 0.56 \\ 70.26 & 0.46\end{array}$

$\begin{array}{ll}70.26 & 0.46 \\ 70.04 & 0.45\end{array}$

$\begin{array}{ll}59.66 & 0.37\end{array}$

$\begin{array}{ll}51.95 & 0.35\end{array}$

$72.14 \quad 0.45$

$\begin{array}{ll}71.68 & 0.45\end{array}$

$\begin{array}{ll}71.58 & 0.43\end{array}$

$\begin{array}{ll}62.57 & 0.40\end{array}$

$\begin{array}{ll}65.51 & 0.43\end{array}$

$\begin{array}{ll}68.81 & 0.49\end{array}$

$\begin{array}{ll}55.34 & 0.27 \\ 66.82 & 0.53\end{array}$

$\begin{array}{ll}66.82 & 0.53 \\ 73.07 & 0.43\end{array}$

$\begin{array}{ll}68.93 & 0.50\end{array}$

$\begin{array}{ll}61.60 & 0.44\end{array}$

$\begin{array}{ll}67.39 & 0.44\end{array}$

$\begin{array}{ll}65.96 & 0.46\end{array}$

$\begin{array}{ll}63.06 & 0.64\end{array}$

$\begin{array}{ll}65.00 & 0.61\end{array}$

$\begin{array}{ll}67.68 & 0.54\end{array}$

$\begin{array}{ll}68.50 & 0.54\end{array}$

$\begin{array}{ll}70.56 & 0.47\end{array}$

$\begin{array}{ll}70.42 & 0.46\end{array}$

$\begin{array}{ll}79.02 & 0.33\end{array}$

$\begin{array}{ll}72.42 & 0.49\end{array}$

$\begin{array}{ll}64.80 & 0.43\end{array}$

$\begin{array}{ll}73.25 & 0.43 \\ 74.83 & 0.35\end{array}$

$68.74 \quad 0.45$

$\begin{array}{ll}66.87 & 0.55\end{array}$

$\begin{array}{ll}69.46 & 0.49 \\ 72.74 & 0.38\end{array}$

$\begin{array}{ll}67.89 & 0.48\end{array}$

$67.11 \quad 0.5$

$\begin{array}{ll}69.10^{\circ} & 0.52\end{array}$

$68.41 \quad 0.51$

$68.91 \quad 0.48$

$\begin{array}{ll}69.05 & 0.52\end{array}$

$\begin{array}{ll}73.62 & 0.42\end{array}$

13.21

13.67
10.82

9.16

10.40

11.31

10.09
10.47

10.24

10.70

13.78
12.63

11.14

12.56

12.86

13.25

12.54

12.53

12.10

\section{Site 434}

$\begin{array}{rr}2.62 & 1.56 \\ 2.72 & 1.72 \\ 2.82 & 1.63 \\ 2.80 & 1.62 \\ 13.87 & 1.82\end{array}$

$\begin{array}{llllllll}0.082 & 1.85 & 2.04 & 3.47 & 2.08 & 0.090 & 4.58\end{array}$

$\begin{array}{lllllll}0.071 & 2.56 & 2.04 & 3.42 & 1.86 & 0.100 & 5.79\end{array}$

$\begin{array}{lllllll}0.060 & 2.26 & 1.60 & 3.61 & 1.92 & 0.076 & 5.30 \\ 0.056 & 2.51 & 1.41 & 3.16 & 2.33 & 0.082 & 6.68\end{array}$

$\begin{array}{lllllll}0.056 & 2.51 & 1.41 & 3.16 & 2.33 & 0.082 & 6.68 \\ 0.250 & 1.83 & 1.87 & 3.09 & 1.70 & 0.077 & 5.94\end{array}$

$\begin{array}{rrr}9.86 & 13.87 & 1.82 \\ 10.31 & 3.26 & 1.39\end{array}$

$\begin{array}{lllll}9.95 & 2.42 & 1.44 & 0.041 & 1.80 \\ 14.18 & 3.32 & 1.77 & 0.053 & 1.95\end{array}$

$\begin{array}{lllll}14.18 & 3.32 & 1.77 & 0.058 & 2.98\end{array}$

$\begin{array}{lll}0.46 & 2.80 \quad 1.20\end{array}$

$\begin{array}{rrr}9.28 & 2.81 & 1.09 \\ 10.19 & 2.50 & 1.72\end{array}$

10.82
10.40

$\begin{array}{rr}10.40 & 2.52 \\ 9.11 & 12.07\end{array}$

$12.07 \quad 1.60$

$\begin{array}{lll}10.32 & 2.67 & 1.33 \\ 0.94 & 2.90 & 1.15\end{array}$

$\begin{array}{lll}10.20 & 2.72 & 1.20\end{array}$

$4.00 \quad 1.28$

$4.28 \quad 1.50$

$3.04 \quad 1.42$

14.31

3.72

$2.30 \quad 1.35$

$2.40 \quad 1.80$

$\begin{array}{ll}8.50 & 1.74\end{array}$

$\begin{array}{ll}4.11 & 2.19 \\ 3.94 & 1.59\end{array}$

0.0612 .08

$0.034 \quad 2.06$

$\begin{array}{ll}0.500 & 1.26\end{array}$

$0.054 \quad 2.42$

$\begin{array}{ll}0.057 & 2.04 \\ 0.049 & 1.83\end{array}$

$\begin{array}{ll}0.049 & 1.83 \\ 0.041 & 1.99\end{array}$

$\begin{array}{ll}0.220 & 2.14\end{array}$

$0.260 \quad 2.03$

$\begin{array}{ll}0.055 & 1.89\end{array}$

$0.057 \quad 1.75$

$\begin{array}{ll}0.045 & 1.72 \\ 0.110 & 2.16\end{array}$

$\begin{array}{ll}0.110 & 1.97\end{array}$

$\begin{array}{ll}0.052 & 2.01\end{array}$

$\begin{array}{ll}0.270 & 2.36 \\ 0.260 & 1.56\end{array}$

$\begin{array}{ll}0.260 & 1.56 \\ 0.060 & 1.57\end{array}$

$0.160 \quad 1.78$

$0.200 \quad 2.25$

$0.120 \quad 1.66$

$\begin{array}{lllll}1.10 & 2.82 & 1.80 & 0.065 & 7.04\end{array}$

$\begin{array}{lllll}1.09 & 2.17 & 1.73 & 0.054 & 5.70\end{array}$

$\begin{array}{lllll}1.03 & 2.35 & 1.70 & 0.058 & 6.18\end{array}$

$\begin{array}{lllll}1.55 & 3.81 & 2.50 & 0.086 & 7.10\end{array}$

$\begin{array}{lllll}1.35 & 3.01 & 1.84 & 0.056 & 6.51 \\ 0.93 & 2.40 & 1.63 & 0.054 & 5.61\end{array}$

$\begin{array}{lllll}1.10 & 2.23 & 1.81 & 0.072 & 4.62\end{array}$

$\begin{array}{lllll}1.85 & 3.08 & 2.29 & 0.085 & 5.24 \\ 2.75 & 3.01 & 1.85 & 0.077 & 5.45\end{array}$

$\begin{array}{lllll}2.75 & 3.01 & 1.85 & 0.077 & 5.45 \\ 1.34 & 2.94 & 1.79 & 0.064 & 5.60\end{array}$

$\begin{array}{lllll}1.08 & 2.53 & 1.83 & 0.058 & 5.79\end{array}$

$\begin{array}{lllll}0.10 & 2.44 & 1.53 & 0.082 & 5.57 \\ 0.40 & 2.43 & 1.35 & 0.130 & 4.31\end{array}$

$\begin{array}{lllll}0.41 & 2.05 & 1.69 & 0.064 & 5.62\end{array}$

$\begin{array}{lllll}1.16 & 2.21 & 1.79 & 0.053 & 5.66\end{array}$

$\begin{array}{lllll}1.02 & 2.35 & 1.74 & 0.054 & 5.72\end{array}$

$\begin{array}{lll}0.80 & 2.19 & 1.67\end{array}$

$\begin{array}{lll}0.12 & 2.56 & 1.74\end{array}$

$\begin{array}{lll}1.16 & 2.55 & 1.93 \\ 0.07 & 2.26 & 1.60\end{array}$

$\begin{array}{lll}0.07 & 2.26 & 1.60 \\ 1.04 & 2.09 & 2.06\end{array}$

$\begin{array}{lll}1.04 & 2.18 & 1.63\end{array}$

$\begin{array}{lll}0.73 & 2.53 & 1.95\end{array}$

$\begin{array}{lll}1.14 & 2.55 & 1.73\end{array}$

$\begin{array}{lll}0.61 & 2.40 & 1.81\end{array}$

$0.110 \quad 1.88$

$0.98 \quad 3.10 \quad 1.84$

$\begin{array}{ll}0.130 & 5.70 \\ 0.086 & 5.56\end{array}$

$0.070 \quad 6.65$

$0.110 \quad 5.10$

$0.110 \quad 5.31$

$\begin{array}{ll}0.067 & 6.02 \\ 0.081 & 4.89\end{array}$

$\begin{array}{ll}0.081 & 4.89 \\ 0.099 & 4.80\end{array}$

$0.100 \quad 5.37$

$0.079 \quad 6.15$

0.0

0.0

0.0
0.0

0.0

0.0

0.0
0.0

0.0

0.0
0.0

0.0
0.0

0.0

0.0

0.0

0.0

5.30

11.52

1.19

0.0

0.0
7.61

4.90

0.0

6.91

1.32

1.09

3.31

2.41
2.17

0.0

$\begin{array}{ll}0.064 & 2.81\end{array}$

$\begin{array}{lll}3.09 & 3.44 & 2.01 \\ 1.75 & 3.51 & 2.30\end{array}$

$0.100 \quad 5.24$

$0.093=5.06$

0.0

$\begin{array}{lllll}1.55 & 2.43 & 2.29 & 0.093 & 5.06 \\ 1.38 & 2.28 & 1.95 & 0.072 & 5.78\end{array}$

$\begin{array}{lll}0.74 & 1.96 & 1.74\end{array}$

10.34
9.48

7.40

10.26
$3.31 \quad 1.51$

$\begin{array}{ll}3.64 & 1.47\end{array}$

$3.11 \quad 1.67$

$\begin{array}{ll}1.46 & 1.33\end{array}$

$\begin{array}{ll}2.36 & 1.71\end{array}$

$\begin{array}{ll}1.82 & 1.24\end{array}$

$3.17 \quad 1.51$

$\begin{array}{ll}3.44 & 1.50 \\ 2.22 & 1.55\end{array}$

$\begin{array}{ll}1.89 & 1.28\end{array}$

$2.72 \quad 1.71$

$3.62 \quad 1.61$

$\begin{array}{ll}3.32 & 1.48 \\ 3.11 & 1.60\end{array}$

$\begin{array}{ll}3.02 & 1.56\end{array}$

$\begin{array}{ll}2.34 & 1.73 \\ 2.94 & 1.76\end{array}$

$2.44 \quad 1.36$
$2.30 \quad 1.51$

$\begin{array}{ll}0.069 & 1.87\end{array}$

$0.048 \quad 1.72$

$\begin{array}{lll}0.130 & 1.63\end{array}$

$0.047 \quad 1.24$

$0.200 \quad 1.50$

$\begin{array}{ll}0.190 & 1.49 \\ 0.041 & 1.34\end{array}$

$\begin{array}{ll}0.036 & 1.17\end{array}$

$\begin{array}{ll}0.120 & 1.87\end{array}$

$\begin{array}{ll}0.110 & 1.92\end{array}$

$\begin{array}{ll}0.042 & 1.70\end{array}$

$\begin{array}{lll}0.130 & 1.54\end{array}$

$\begin{array}{lll}0.150 & 1.89\end{array}$

$\begin{array}{ll}0.041 & 1.86\end{array}$

$\begin{array}{ll}0.300 & 1.59 \\ 0.100 & 1.77\end{array}$

$\begin{array}{ll}0.099 & 1.62\end{array}$

$\begin{array}{ll}0.086 & 2.01 \\ 0.035 & 1.65\end{array}$

$\begin{array}{lll}0.20 & 2.13 & 1.58\end{array}$

$\begin{array}{lll}0.86 & 1.64 & 1.29 \\ 0.38 & 2.21 & 1.66\end{array}$

$\begin{array}{lll}0.64 & 2.01 & 1.83\end{array}$

$\begin{array}{lll}1.00 & 1.92 & 1.73\end{array}$

$\begin{array}{lll}1.29 & 2.53 & 1.70\end{array}$

$\begin{array}{lll}0.79 & 2.22 & 1.90 \\ 1.03 & 2.05 & 2.66\end{array}$

$\begin{array}{lll}1.03 & 2.05 & 2.66 \\ 1.38 & 2.51 & 2.06\end{array}$

$\begin{array}{lll}1.47 & 2.54 & 1.68\end{array}$

$\begin{array}{lll}0.15 & 2.17 & 2.30\end{array}$

$\begin{array}{lll}0.85 & 2.25 & 2.38\end{array}$

$\begin{array}{lll}0.62 & 2.01 & 2.25 \\ 0.44 & 2.18 & 2.28\end{array}$

$\begin{array}{lll}0.44 & 2.18 & 2.28 \\ 0.62 & 2.29 & 2.36\end{array}$

$\begin{array}{lll}0.62 & 2.29 & 2.36 \\ 0.81 & 2.28 & 2.24\end{array}$ 
TABLE 1 - Continued

Sample

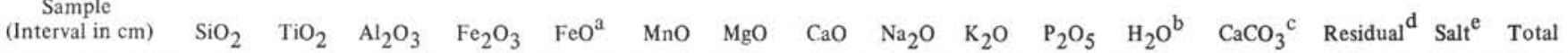

Hole 435A

\begin{tabular}{|c|c|c|c|c|c|c|c|c|c|c|c|c|c|c|c|c|}
\hline $1-3,54-58$ & 61.45 & 0.64 & 13.91 & 3.05 & 2.12 & 0.065 & 2.55 & 2.58 & 3.45 & 2.07 & 0.110 & 5.92 & 1.24 & 0.57 & 0.42 & 100.15 \\
\hline $2-1,43-47$ & 73.80 & 0.32 & 6.86 & 1.81 & 1.03 & 0.029 & 1.33 & 1.91 & 2.53 & 1.16 & 0.064 & 7.05 & 0.0 & 1.25 & 0.70 & 99.84 \\
\hline $3-2,66-70$ & 74.33 & 0.29 & 6.70 & 1.49 & 0.97 & 0.048 & 1.17 & 1.20 & 2.43 & 1.14 & 0.059 & 6.13 & 0.0 & 2.75 & 0.67 & 99.38 \\
\hline $4-2,50-54$ & 72.29 & 0.38 & 8.39 & 1.88 & 1.34 & 0.041 & 1.71 & 1.19 & 2.38 & 1.41 & 0.056 & 6.68 & 0.0 & 2.00 & 0.61 & 100.36 \\
\hline $5-3,90-94$ & 69.26 & 0.42 & 9.79 & 2.24 & 1.36 & 0.042 & 1.59 & 1.72 & 2.52 & 1.57 & 0.075 & 6.56 & 1.17 & 0.49 & 0.44 & 99.25 \\
\hline $6-1,58-62$ & 69.47 & 0.37 & 8.38 & 2.45 & 1.12 & 0.034 & 1.27 & 1.25 & 2.23 & 1.32 & 0.069 & 6.87 & 4.09 & 0.17 & 0.49 & 99.57 \\
\hline $7-1,46-50$ & 72.29 & 0.38 & 8.52 & 2.06 & 1.31 & 0.030 & 1.68 & 1.18 & 2.37 & 1.39 & 0.065 & 8.68 & 0.0 & 0.42 & 0.56 & 100.94 \\
\hline $8-1,43-47$ & 77.59 & 0.27 & 5.41 & 1.40 & 0.89 & 0.021 & 1.22 & 1.03 & 2.18 & 0.89 & 0.058 & 7.53 & 0.0 & 1.48 & 0.71 & 100.67 \\
\hline $9-1,110-114$ & 73.31 & 0.33 & 8.01 & 1.84 & 1.05 & 0.026 & 1.44 & 1.02 & 2.45 & 1.42 & 0.049 & 7.15 & 0.0 & 1.54 & 0.63 & 100.27 \\
\hline $10-1,62-66$ & 72.55 & 0.41 & 9.33 & 2.54 & 1.19 & 0.045 & 1.89 & 1.37 & 2.27 & 1.51 & 0.068 & 6.72 & 0.0 & 0.66 & 0.43 & 100.98 \\
\hline $11-2,66-70$ & 69.97 & 0.34 & 7.83 & 2.14 & 1.05 & 0.046 & 1.45 & 0.90 & 2.09 & 1.31 & 0.094 & 6.70 & 3.71 & 1.15 & 0.48 & 99.25 \\
\hline
\end{tabular}

Site 436

Hole 436

\begin{tabular}{llll}
$1-1,50-54$ & 60.98 & 0.64 & 14.16 \\
$1-4,50-54$ & 61.69 & 0.55 & 13.48 \\
$2-1,110-114$ & 62.04 & 0.63 & 13.79 \\
$3-3,100-104$ & 62.16 & 0.57 & 13.78 \\
$4-2,130-134$ & 62.69 & 0.56 & 12.90 \\
$4-4,130-134$ & 60.95 & 0.61 & 13.91 \\
$5-2,14-18$ & 60.41 & 0.61 & 14.40 \\
$5-4,14-18$ & 61.77 & 0.61 & 14.06 \\
$6-3,80-84$ & 62.18 & 0.63 & 14.76 \\
$6-3,84-88$ & 64.39 & 0.55 & 13.88 \\
$7-6,6-10$ & 61.64 & 0.63 & 14.39 \\
$8-2,50-54$ & 63.23 & 0.55 & 13.47 \\
$8-4,50-54$ & 62.27 & 0.57 & 13.22 \\
$9-3,113-118$ & 64.86 & 0.48 & 12.39 \\
$9-6,41-46$ & 60.33 & 0.64 & 14.32 \\
$10-2,113-118$ & 63.92 & 0.54 & 12.60 \\
$10-5,113-118$ & 62.56 & 0.49 & 13.13 \\
$11-2,86-90$ & 62.50 & 0.52 & 12.10 \\
$11-5,86-90$ & 62.32 & 0.52 & 14.10 \\
$12-2,36-40$ & 64.61 & 0.53 & 13.28 \\
$12-4,36-40$ & 67.36 & 0.33 & 12.27 \\
$13-2,73-75$ & 61.19 & 0.50 & 11.81 \\
$13-4,73-75$ & 63.00 & 0.49 & 11.88 \\
$14-1,60-64$ & 66.06 & 0.35 & 12.14 \\
$14-3,60-64$ & 69.58 & 0.31 & 12.25 \\
$15-1,90-94$ & 67.57 & 0.46 & 11.60 \\
$15-6,30-34$ & 68.59 & 0.30 & 11.98 \\
$16-2,90-94$ & 69.66 & 0.41 & 10.27 \\
$17-4,30-34$ & 64.55 & 0.56 & 12.49 \\
$18-2,34-38$ & 64.35 & 0.46 & 11.71 \\
$19-1,66-70$ & 64.37 & 0.44 & 12.02 \\
$19-4,86-90$ & 64.57 & 0.47 & 12.04 \\
$20-1,105-109$ & 67.82 & 0.41 & 12.21 \\
$21-2,64-68$ & 64.57 & 0.56 & 13.86 \\
$22-1,52-56$ & 62.10 & 0.53 & 13.04 \\
$23-2,35-39$ & 64.23 & 0.56 & 13.62 \\
$23-5,35-39$ & 64.42 & 0.55 & 13.53 \\
$24-1,53-57$ & 64.55 & 0.56 & 14.13 \\
$25-1,74-78$ & 65.27 & 0.49 & 13.14 \\
$27-1,106-110$ & 64.29 & 0.56 & 14.76 \\
$27-2,106-110$ & 62.59 & 0.56 & 13.50 \\
$28-1,56-60$ & 65.00 & 0.54 & 13.95 \\
$29-1,70-74$ & 67.45 & 0.44 & 13.55 \\
$29-2,70-74$ & 66.05 & 0.39 & 13.10 \\
$30-1,74-78$ & 66.28 & 0.38 & 12.98 \\
$30-3,74-78$ & 64.03 & 0.53 & 13.97 \\
$31-2,40-44$ & 64.41 & 0.42 & 12.10 \\
$31-4,40-44$ & 61.95 & 0.58 & 14.76 \\
$32-2,46-50$ & 61.90 & 0.55 & 13.42 \\
$33-1,52-56$ & 63.46 & 0.48 & 13.67 \\
$33-4,52-56$ & 60.51 & 0.58 & 14.42 \\
$34-2,60-64$ & 62.02 & 0.59 & 14.09 \\
$34-6,60-64$ & 63.64 & 0.55 & 13.72 \\
$35-1,60-64$ & 60.36 & 0.58 & 14.01 \\
$35-4,60-64$ & 58.57 & 0.65 & 15.18 \\
$37-60-50-74$ & 62.02 & 0.62 & 15.86 \\
\hline & 62.76 & 0.53 & 15.42 \\
36.07 & 0.59 & 15.30 \\
30.88 & 0.45 & 16.44
\end{tabular}

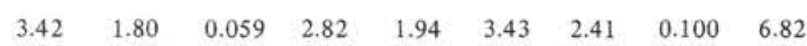

$\begin{array}{lllllllll}4.27 & 1.68 & 0.081 & 1.95 & 2.15 & 3.36 & 2.19 & 0.092 & 6.25\end{array}$

$\begin{array}{lllllll}3.99 & 2.29 & 0.083 & 2.47 & 2.41 & 3.29 & 2.16\end{array}$

$\begin{array}{lllllll}3.20 & 1.69 & 0.056 & 2.31 & 1.41 & 3.47 & 2.61\end{array}$

$3.70 \quad 1.52$

$3.71 \quad 1.74$

$\begin{array}{ll}3.30 & 1.92 \\ 3.85 & 1.83\end{array}$

$\begin{array}{lllllll}3.32 & 1.90 & 0.063 & 2.74 & 2.11 & 3.26 & 2.46\end{array}$

$\begin{array}{lllllll}2.83 & 1.73 & 0.066 & 2.44 & 1.89 & 3.56 & 2.32\end{array}$

$3.48 \quad 1.73$

$3.03 \quad 1.68$

$3.24 \quad 1.72$

$\begin{array}{ll}3.45 & 1.41 \\ 3.77 & 1.57\end{array}$

$\begin{array}{lllllll}3.77 & 1.57 & 0.055 & 3.19 & 1.44 & 3.20 & 2.69\end{array}$

$\begin{array}{lllllll}3.30 & 1.40 & 0.058 & 2.59 & 1.37 & 3.38 & 2.26\end{array}$

$\begin{array}{lllllll}3.66 & 1.66 & 0.074 & 2.51 & 1.66 & 3.63 & 2.21\end{array}$

$\begin{array}{lllllll}3.58 & 1.47 & 0.061 & 2.81 & 1.59 & 3.76 & 2.13\end{array}$

$\begin{array}{lllllll}3.25 & 1.73 & 0.071 & 2.24 & 2.36 & 3.65 & 2.07 \\ 1.74 & 1.20 & 0.063 & 2.31 & 1.74 & 3.87 & 2.22\end{array}$

$\begin{array}{lllllll}1.74 & 1.18 & 0.073 & 1.49 & 1.21 & 3.37 & 2.98\end{array}$

$\begin{array}{lllllll}4.35 & 1.47 & 0.072 & 2.51 & 1.59 & 4.00 & 2.02\end{array}$

$\begin{array}{lllllll}2.80 & 1.49 & 0.060 & 2.28 & 1.63 & 3.63 & 2.14\end{array}$

$\begin{array}{lllllll}3.31 & 1.30 & 0.073 & 1.61 & 1.16 & 3.49 & 3.14 \\ 1.68 & 1.02 & 0.051 & 1.13 & 1.59 & 3.47 & 2.88\end{array}$

$\begin{array}{lllllll}2.41 & 1.43 & 0.050 & 2.16 & 1.81 & 3.61 & 1.92\end{array}$

$\begin{array}{lllllll}1.53 & 1.09 & 0.058 & 1.27 & 1.27 & 3.35 & 3.18 \\ 2.85 & 1.26 & 0.048 & 1.95 & 1.32 & 3.19 & 1.75\end{array}$

$\begin{array}{lllllll}2.85 & 1.26 & 0.048 & 1.95 & 1.32 & 3.19 & 1.75 \\ 2.79 & 1.69 & 0.061 & 1.95 & 2.02 & 3.41 & 1.95\end{array}$

$\begin{array}{lllllll}3.42 & 1.43 & 0.062 & 1.91 & 1.67 & 3.69 & 2.06\end{array}$

$\begin{array}{lllllll}3.09 & 1.26 & 0.066 & 1.66 & 1.82 & 3.70 & 2.31 \\ 3.82 & 1.21 & 0.044 & 2.07 & 1.84 & 3.26 & 1.93\end{array}$

$\begin{array}{lllllll}3.82 & 1.21 & 0.044 & 2.07 & 1.84 & 3.26 & 1.93 \\ 2.11 & 1.59 & 0.057 & 1.46 & 1.60 & 3.42 & 2.11\end{array}$

$\begin{array}{lllllll}2.68 & 1.60 & 0.053 & 2.401 & 1.60 & 3.42 & 2.11\end{array}$

$\begin{array}{lllllll}4.04 & 1.49 & 0.055 & 2.50 & 1.27 & 3.34 & 2.58 \\ 3.17 & 1.39 & 0.043 & 2.19 & 1.54 & 3.36 & 2.43\end{array}$

$\begin{array}{lllllll}3.17 & 1.39 & 0.043 & 2.19 & 1.54 & 3.36 & 2.43 \\ 2.75 & 1.70 & 0.058 & 2.20 & 1.52 & 3.31 & 2.55\end{array}$

$\begin{array}{lllllll}3.36 & 1.35 & 0.061 & 2.18 & 1.24 & 3.16 & 2.64\end{array}$

$\begin{array}{lllllll}3.46 & 1.14 & 0.050 & 1.94 & 1.06 & 3.03 & 2.67\end{array}$

$3.59 \quad 1.36$

$\begin{array}{ll}4.17 & 1.48\end{array}$

$\begin{array}{ll}3.45 & 1.19 \\ 3.20 & 1.22\end{array}$

$\begin{array}{ll}3.20 & 1.22 \\ 3.28 & 0.81\end{array}$

$2.45 \quad 0.95$

$\begin{array}{ll}3.66 & 1.05\end{array}$

$\begin{array}{ll}3.52 & 0.95 \\ 4.40 & 0.99\end{array}$

$4.40 \quad 0.99$

$\begin{array}{ll}4.59 & 0.80 \\ 3.82 & 0.90\end{array}$

$\begin{array}{ll}3.82 & 0.90 \\ 4.65 & 1.02\end{array}$

$4.66 \quad 0.99$

$4.77 \quad 0.80$

4.68
5.25

$5.25 \quad 0.88$

$\begin{array}{lllll}0.450 & 1.61 & 0.80 & 2.56 & 2.81\end{array}$

$\begin{array}{lllllll}5.17 & 0.68 & 0.240 & 1.42 & 0.87 & 3.04 & 3.21 \\ 4.02 & 0.32 & 0.370 & 1.84 & 0.70 & 2.65 & 2.86\end{array}$

$\begin{array}{ll}4.02 & 0.32\end{array}$ $\begin{array}{lllll}0.048 & 2.16 & 1.01 & 3.03 & 2.70\end{array}$

$\begin{array}{lllll}0.041 & 2.49 & 1.04 & 3.14 & 2.57 \\ 0.040 & 2.19 & 0.87 & 3.15 & 2.57\end{array}$

$\begin{array}{lllll}0.078 & 1.58 & 1.56 & 3.26 & 2.45 \\ 0.067 & 1.71 & 0.97 & 3.05 & 3.09\end{array}$

$\begin{array}{lllll}0.067 & 1.71 & 0.97 & 3.05 & 3.09 \\ 0.120 & 1.38 & 1.05 & 3.00 & 3.13\end{array}$

$\begin{array}{lllll}0.055 & 2.09 & 1.18 & 2.88 & 2.56\end{array}$

$\begin{array}{lllll}0.180 & 1.69 & 1.38 & 3.09 & 2.31 \\ 0.650 & 1.40 & 1.29 & 3.20 & 2.51\end{array}$

$\begin{array}{lllll}0.560 & 1.46 & 0.96 & 3.08 & 2.57\end{array}$

$\begin{array}{lllll}0.350 & 1.43 & 1.10 & 3.16 & 2.78\end{array}$

$\begin{array}{lllll}0.310 & 2.04 & 1.31 & 3.23 & 2.55\end{array}$

$\begin{array}{lllll}0.180 & 2.24 & 1.26 & 2.96 & 2.49 \\ 0.170 & 2.11 & 1.07 & 3.10 & 2.49\end{array}$

$\begin{array}{lllll}0.170 & 2.11 & 1.07 & 3.10 & 2.49 \\ 0.900 & 0.83 & 0.78 & 3.51 & 2.75\end{array}$

$\begin{array}{ll}0.370 & 2.51\end{array}$ $\begin{array}{ll}0.100 & 6.35 \\ 0.083 & 7.21\end{array}$

$\begin{array}{ll}0.083 & 7.21 \\ 0.083 & 7.33\end{array}$

$\begin{array}{ll}0.083 & 7.33 \\ 0.088 & 6.90\end{array}$

$0.100 \quad 6.17$

$0.100 \quad 4.50$

$\begin{array}{ll}0.085 & 6.43 \\ 0.100 & 6.06\end{array}$

$0.093 \quad 5.96$

$\begin{array}{ll}0.089 & 6.45 \\ 0.068 & 6.58\end{array}$

$0.089 \quad 6.95$

$0.074 \quad 5.97$

$\begin{array}{ll}0.076 & 5.81 \\ 0.072 & 6.67\end{array}$

$0.110 \quad 5.52$

$\begin{array}{ll}0.058 & 6.04 \\ 0.074 & 6.58\end{array}$

$0.074 \quad 5.57$

$\begin{array}{ll}0.047 & 4.76 \\ 0.085 & 6.91\end{array}$

$\begin{array}{ll}0.050 & 5.67\end{array}$

$0.062 \quad 7.30$

$\begin{array}{ll}0.090 & 5.87 \\ 0.062 & 6.98\end{array}$

$\begin{array}{ll}0.120 & 6.34\end{array}$

$0.057 \quad 5.68$

$\begin{array}{ll}0.062 & 5.90 \\ 0.088 & 5.90\end{array}$

$\begin{array}{ll}0.064 & 7.07\end{array}$

$0.064 \quad 6.63$

$0.082 \quad 6.87$

$\begin{array}{ll}0.076 & 6.34\end{array}$

$\begin{array}{ll}0.063 & 6.20 \\ 0.063 & 6.69\end{array}$

$0.061 \quad 6.50$

$\begin{array}{ll}0.068 & 6.72\end{array}$

$\begin{array}{ll}0.064 & 5.66 \\ 0.057 & 5.29\end{array}$

$0.055 \quad 5.15$

$0.065 \quad 3.85$

$\begin{array}{ll}0.170 & 6.50\end{array}$

$0.077 \quad 6.04$

$\begin{array}{ll}0.070 & 7.08 \\ 0.110 & 6.66\end{array}$

$\begin{array}{ll}0.110 & 6.66 \\ 0.092 & 8.34\end{array}$

$0.083-6.23$

$0.074 \quad 6.00$

$\begin{array}{ll}0.080 & 8.19\end{array}$

$0.090 \quad 7.95$

$\begin{array}{ll}0.097 & 6.89 \\ 0.074 & 6.44\end{array}$
$0.097 \quad 6.25$

$0.088 \quad 5.51$

$0.046 \quad 4.24$ $\begin{array}{llll}0.0 & 1.73 & 0.64 & 100.94\end{array}$

$\begin{array}{llll}0.0 & 1.11 & 0.51 & 99.36\end{array}$

$\begin{array}{llll}0.0 & 0.34 & 0.55 & 100.50\end{array}$

$\begin{array}{lllr}0.0 & 0.44 & 0.56 & 99.55 \\ 0.0 & 0.68 & 0.75 & 100.80\end{array}$

$\begin{array}{llll}0.0 & 0.68 & 0.75 & 100.80 \\ 0.0 & 0.88 & 0.60 & 99.72\end{array}$

$\begin{array}{llll}0.0 & 0.88 & 0.60 & 99.72 \\ 0.0 & 0.84 & 0.53 & 99.21\end{array}$

$\begin{array}{llll}0.0 & 1.38 & 0.53 & 100.67\end{array}$

$\begin{array}{llll}0.0 & 2.46 & 0.50 & 101.00\end{array}$

$\begin{array}{llll}0.0 & 0.29 & 0.48 & 100.93\end{array}$

$\begin{array}{llll}0.0 & 0.33 & 0.54 & 99.62\end{array}$

$\begin{array}{llll}0.0 & 0.41 & 0.58 & 99.45 \\ 0.0 & 0.58 & 0.58 & 99.27\end{array}$

$\begin{array}{lllr}0.0 & 0.28 & 0.65 & 100.10\end{array}$

$\begin{array}{llll}0.0 & 0.97 & 0.58 & 99.78\end{array}$

$\begin{array}{llll}0.0 & 1.65 & 0.74 & 99.86\end{array}$

$\begin{array}{llll}0.0 & 1.24 & 0.55 & 99.26\end{array}$

$\begin{array}{llll}0.0 & 1.86 & 0.81 & 99.92 \\ 0.0 & 1.01 & 0.58 & 99.28\end{array}$

$\begin{array}{lllll}0.0 & 1.69 & 0.64 & 100.99\end{array}$

$\begin{array}{llll}0.0 & 0.72 & 0.63 & 99.45\end{array}$

$\begin{array}{llll}0.0 & 2.19 & 0.71 & 99.06\end{array}$

$\begin{array}{llll}0.0 & 3.18 & 0.67 & 98.90\end{array}$

$\begin{array}{llll}0.0 & 2.32 & 0.56 & 99.79\end{array}$

$\begin{array}{llll}0.0 & 0.17 & 0.38 & 99.31\end{array}$

$\begin{array}{llll}0.0 & 0.22 & 0.74 & 100.98\end{array}$

$\begin{array}{llll}0.0 & 0.81 & 0.49 & 99.64\end{array}$

$\begin{array}{lllll}0.0 & 0.17 & 0.64 & 100.88\end{array}$

$\begin{array}{rrrr}0.0 & 1.04 & 0.65 & 99.12 \\ 0.0 & 1.62 & 0.72 & 100.15\end{array}$

$\begin{array}{llll}0.0 & 1.22 & 0.70 & 99.12\end{array}$

$\begin{array}{llll}0.0 & 1.50 & 0.64 & 99.12\end{array}$

$\begin{array}{llll}0.0 & 0.12 & 0.51 & 99.38\end{array}$

$\begin{array}{llll}0.0 & 0.58 & 0.59 & 99.75\end{array}$

$\begin{array}{llll}0.0 & 0.43 & 0.61 & 99.13\end{array}$

$\begin{array}{llll}0.0 & 0.11 & 0.64 & 99.97\end{array}$

$\begin{array}{llll}0.0 & 0.06 & 0.56 & 100.16\end{array}$

$\begin{array}{llll}0.0 & 0.71 & 0.61 & 100.97\end{array}$

$\begin{array}{llll}0.0 & 0.54 & 0.63 & 99.69\end{array}$

$\begin{array}{llll}0.0 & 0.01 & 0.70 & 100.97\end{array}$

$\begin{array}{llll}0.0 & 0.75 & 0.59 & 99.47\end{array}$

$\begin{array}{llll}0.0 & 0.39 & 0.64 & 100.76\end{array}$

$\begin{array}{llll}0.0 & 0.08 & 0.50 & 101.11\end{array}$

$\begin{array}{llll}0.0 & 1.80 & 0.54 & 100.20\end{array}$

$\begin{array}{llll}0.0 & 1.74 & 0.39 & 99.07\end{array}$

$\begin{array}{llll}0.0 & 2.56 & 0.55 & 99.04\end{array}$

$\begin{array}{llll}0.0 & 2.33 & 0.54 & 99.59\end{array}$

$\begin{array}{llll}0.0 & 1.80 & 0.51 & 100.16\end{array}$

$\begin{array}{llll}0.0 & 2.28 & 0.49 & 99.81\end{array}$

$\begin{array}{llll}0.0 & 1.88 & 0.47 & 100.26\end{array}$

$\begin{array}{llll}0.0 & 1.13 & 0.48 & 100.65\end{array}$

$\begin{array}{llll}0.0 & 0.86 & 0.50 & 99.17\end{array}$

$\begin{array}{llll}0.0 & 1.35 & 0.49 & 100.33\end{array}$

$\begin{array}{llll}0.0 & 2.39 & 0.58 & 99.02\end{array}$

$\begin{array}{llll}0.0 & 1.84 & 0.53 & 100.44\end{array}$

$\begin{array}{llll}0.0 & 1.93 & 0.39 & 100.95\end{array}$

$\begin{array}{llll}0.0 & 0.32 & 0.42 & 101.13 \\ 0.0 & 0.76 & 0.47 & 100.35\end{array}$

$\begin{array}{lllr}0.0 & 0.76 & 0.47 & 100.35 \\ 0.0 & 1.34 & 0.31 & 99.01\end{array}$ 
TABLE 1 - Continued

Sample

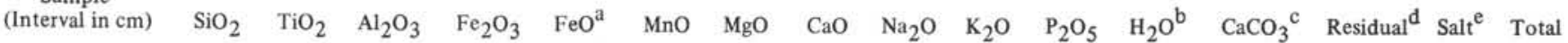

\begin{tabular}{|c|c|c|c|c|c|c|c|c|c|c|c|c|c|c|c|c|}
\hline \multicolumn{17}{|l|}{ 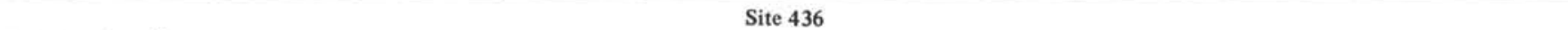 } \\
\hline Hole 436 (cont.) & & & & & & & & & & & & & & & & \\
\hline $38-2,66-70$ & 62.26 & 0.53 & 15.50 & 5.04 & 0.34 & 0.520 & 1.63 & 0.95 & 3.05 & 3.11 & 0.120 & 7.03 & 0.0 & 0.77 & 0.29 & 101.15 \\
\hline $39-1,60-64$ & 58.61 & 0.60 & 16.46 & 6.63 & 0.11 & 0.680 & 1.80 & 0.87 & 2.70 & 2.44 & 0.150 & 9.05 & 0.0 & 1.03 & 0.23 & 101.36 \\
\hline $39-6,60-64$ & 56.84 & 0.67 & 18.58 & 6.89 & 0.09 & 2.140 & 0.18 & 0.68 & 2.42 & 3.15 & 0.320 & 8.69 & 0.0 & 0.15 & 0.23 & 101.03 \\
\hline $40-3,50-54$ & 55.50 & 0.67 & 18.62 & 6.85 & 0.10 & 2.140 & 0.18 & 0.58 & 2.07 & 3.74 & 0.280 & 7.80 & 0.0 & 0.52 & 0.25 & 99.29 \\
\hline $40-4,50-54$ & 56.50 & 0.67 & 18.67 & 6.81 & 0.24 & 2.320 & 0.18 & 0.63 & 1.99 & 3.86 & 0.300 & 7.79 & 0.0 & 0.56 & 0.23 & 100.75 \\
\hline
\end{tabular}

Site 438

Hole 438

\begin{tabular}{|c|c|c|c|c|c|c|c|c|c|c|c|c|c|c|c|c|}
\hline $1-2,98-102$ & 64.65 & 0.43 & 12.90 & 2.42 & 1.77 & 0.068 & 1.55 & 2.31 & 3.02 & 1.98 & 0.076 & 4.07 & 1.83 & 0.70 & 1.29 & 99.07 \\
\hline $3-2,60-64$ & 58.66 & 0.57 & 11.92 & 2.88 & 1.95 & 0.050 & 2.27 & 2.80 & 2.17 & 2.06 & 0.100 & 5.17 & 4.75 & 2.20 & 1.58 & 99.13 \\
\hline $5-4,30-34$ & 61.44 & 0.55 & 12.61 & 5.41 & 1.73 & 0.088 & 2.61 & 3.37 & 2.86 & 1.99 & 0.092 & 4.30 & 0.0 & 1.11 & 1.02 & 99.17 \\
\hline $7-2,60-64$ & 65.53 & 0.47 & 10.85 & 2.33 & 1.72 & 0.059 & 1.90 & 2.22 & 2.38 & 1.67 & 0.092 & 7.12 & 0.0 & 1.42 & 1.64 & 99.40 \\
\hline $9-2,132-136$ & 69.69 & 0.45 & 11.43 & 2.26 & 1.51 & 0.047 & 1.73 & 1.90 & 2.27 & 1.90 & 0.086 & 5.94 & 0.0 & 0.75 & 1.34 & 101.30 \\
\hline $10-3,100-104$ & 64.52 & 0.52 & 11.63 & 2.67 & 1.93 & 0.064 & 2.07 & 2.92 & 2.36 & 1.62 & 0.100 & 5.07 & 0.0 & 1.98 & 1.43 & 98.89 \\
\hline $11-2,76-80$ & 65.39 & 0.52 & 10.93 & 2.99 & 1.76 & 0.056 & 1.78 & 2.68 & 2.38 & 1.68 & 0.092 & 6.19 & 0.0 & 1.49 & 1.25 & 99.18 \\
\hline \multicolumn{17}{|l|}{ lole 438A } \\
\hline $1-2,66-70$ & 58.74 & 0.59 & 13.44 & 4.76 & 1.80 & 0.083 & 2.27 & 3.57 & 2.74 & 1.76 & 0.110 & 5.23 & 1.81 & 1.14 & 1.45 & 99.4 \\
\hline $3-2,72-76$ & 61.57 & 0.52 & 12.71 & 4.10 & 1.69 & 0.067 & 2.31 & 1.91 & 2.82 & 2.12 & 0.091 & 5.38 & 2.70 & 0.40 & 1.29 & 99.68 \\
\hline $5-2,145-147$ & 62.94 & 0.42 & 11.55 & 2.04 & 1.38 & 0.051 & 1.43 & 1.38 & 2.69 & 1.77 & 0.090 & 5.29 & 4.87 & 1.84 & 1.05 & 98.79 \\
\hline $7-2,41-42$ & 65.16 & 0.43 & 8.92 & 2.08 & 1.49 & 0.030 & 1.64 & 0.24 & 1.66 & 1.50 & 0.078 & 10.20 & 3.30 & 0.59 & 1.75 & 99.0 \\
\hline $9-1,22-24$ & 69.59 & 0.40 & 8.02 & 1.92 & 1.30 & 0.034 & 1.37 & 1.01 & 1.87 & 1.30 & 0.065 & 8.51 & 1.74 & 0.23 & 2.02 & 99.3 \\
\hline $11-2,84-88$ & 70.02 & 0.33 & 6.53 & 1.64 & 1.08 & 0.030 & 1.19 & 0.55 & 1.91 & 1.02 & 0.064 & 9.35 & 2.70 & 0.41 & 2.22 & 99.0 \\
\hline $13-2,9-13$ & 70.86 & 0.32 & 6.47 & 1.49 & 1.17 & 0.029 & 1.21 & 1.90 & 1.51 & 1.00 & 0.064 & 8.72 & 0.0 & 1.86 & 2.68 & 99.2 \\
\hline $17-2,62-64$ & 66.22 & 0.47 & 9.89 & 2.27 & 1.72 & 0.040 & 1.89 & 1.19 & 1.74 & 1.67 & 0.077 & 9.00 & 0.0 & 1.40 & 1.62 & 99.2 \\
\hline $19-2,60-64$ & 68.97 & 0.41 & 8.95 & 1.98 & 1.58 & 0.039 & 1.60 & 1.45 & 1.84 & 1.58 & 0.066 & 8.02 & 0.0 & 0.49 & 1.96 & 98.9 \\
\hline $21-2,40-44$ & 68.87 & 0.45 & 9.31 & 2.20 & 1.70 & 0.037 & 1.80 & 1.09 & 1.93 & 1.58 & 0.074 & 8.34 & 0.0 & 0.11 & 2.04 & 99.52 \\
\hline $23-1,34-38$ & 68.88 & 0.39 & 8.06 & 1.98 & 1.44 & 0.031 & 1.39 & 0.97 & 1.58 & 1.37 & 0.062 & 10.92 & 0.0 & 1.90 & 1.68 & 100.65 \\
\hline $24-2,20-22$ & 68.44 & 0.47 & 9.64 & 2.54 & 1.55 & 0.035 & 1.72 & 1.06 & 1.99 & 1.64 & 0.077 & 7.90 & 0.0 & 0.07 & 1.66 & 98.7 \\
\hline $25-2,120-124$ & 66.56 & 0.44 & 9.73 & 2.33 & 1.48 & 0.039 & 1.60 & 1.88 & 1.80 & 1.61 & 0.069 & 10.27 & 0.0 & 0.94 & 1.67 & 100.41 \\
\hline $27-2,50-54$ & 71.56 & 0.37 & 7.70 & 1.76 & 1.24 & 0.028 & 1.39 & 1.18 & 1.34 & 1.32 & 0.063 & 9.40 & 0.0 & 0.26 & 1.83 & 99.4 \\
\hline $29-2$ & 70.43 & 0.30 & 6.15 & 1.82 & 0.92 & 0.017 & 1.18 & 0.66 & 1.39 & 1.08 & 0.045 & 11.76 & 0.0 & 2.98 & 2.00 & 100.7 \\
\hline $31-2,40-44$ & 70.26 & 0.30 & 6.29 & 1.69 & 0.94 & 0.024 & 1.01 & -0.16 & 1.15 & 1.09 & 0.072 & 7.84 & 6.28 & 1.91 & 2.00 & 100.6 \\
\hline $33-2,36-46$ & 67.70 & 0.40 & 9.51 & 2.68 & 1.37 & 0.057 & 1.19 & 2.54 & 1.91 & 1.40 & 0.082 & 8.64 & 0.0 & 2.45 & 1.34 & 101.2 \\
\hline $35-2,10-14$ & 69.96 & 0.33 & 10.10 & 1.75 & 1.49 & 0.050 & 1.29 & 1.50 & 2.22 & 1.91 & 0.066 & 7.35 & 0.0 & 0.33 & 1.25 & 99.5 \\
\hline $39-2,100-102$ & 69.43 & 0.38 & 8.34 & 2.01 & 1.33 & 0.030 & 1.25 & 0.75 & 1.39 & 1.48 & 0.700 & 7.83 & 3.67 & 0.21 & 1.36 & 100.16 \\
\hline $41-2,121-123$ & 69.71 & 0.40 & 8.78 & 2.15 & 1.26 & 0.028 & 1.39 & 1.38 & 1.66 & 1.56 & 0.064 & 8.49 & 0.0 & 0.47 & 1.69 & 99.02 \\
\hline $43-2,132-136$ & 67.69 & 0.47 & 10.59 & 2.77 & 1.65 & 0.040 & 1.72 & 1.34 & 1.97 & 1.88 & 0.067 & 6.61 & 0.0 & 0.99 & 0.99 & 98.78 \\
\hline $47-2,72-76$ & 72.26 & 0.45 & 9.66 & 2.26 & 1.47 & 0.028 & 1.87 & 0.82 & 1.59 & 1.69 & 0.070 & 7.62 & 0.0 & 0.05 & 1.14 & 100.97 \\
\hline $49-2,80-82$ & 68.90 & 0.45 & 9.54 & 2.13 & 1.72 & 0.039 & 1.87 & 0.99 & 1.61 & 1.72 & 0.074 & 8.16 & 0.0 & 0.83 & 0.93 & 98.96 \\
\hline $51-2,27-29$ & 65.28 & 0.54 & 11.91 & 2.46 & 2.06 & 0.044 & 2.30 & 1.60 & 2.13 & 2.20 & 0.081 & 7.98 & 0.0 & 1.54 & 0.82 & 100.94 \\
\hline $53-1,116-120$ & 65.38 & 0.41 & 8.39 & 2.07 & 1.47 & 0.027 & 1.73 & 1.00 & 1.74 & 1.57 & 0.063 & 12.25 & 0.0 & 3.70 & 1.05 & 100.85 \\
\hline $55-2,30-32$ & 65.42 & 0.39 & 7.93 & 1.88 & 1.34 & 0.026 & 1.47 & 0.68 & 1.63 & 1.47 & 0.059 & 12.39 & 0.0 & 4.84 & 1.13 & 100.65 \\
\hline $57-2,20-24$ & 69.86 & 0.47 & 10.11 & 2.33 & 1.72 & 0.035 & 2.04 & 1.03 & 2.06 & 1.85 & 0.073 & 7.57 & 0.0 & 0.07 & 1.01 & 100.21 \\
\hline $59-2,74-78$ & 67.64 & 0.45 & 9.90 & 2.59 & 1.59 & 0.030 & 2.08 & 1.64 & 1.88 & 1.93 & 0.072 & 7.28 & 0.0 & 2.25 & 1.07 & 100.41 \\
\hline $63-1,42-44$ & 70.66 & 0.39 & 8.08 & 1.94 & 1.32 & 0.042 & 1.57 & 0.68 & 1.35 & 1.42 & 0.063 & 7.23 & 2.66 & 0.23 & 1.43 & 99.07 \\
\hline $65-1,77-79$ & 71.26 & 0.37 & 8.11 & 2.07 & 1.18 & 0.024 & 1.54 & 0.67 & 1.47 & 1.59 & 0.049 & 8.64 & 0.0 & 0.87 & 1.23 & 99.06 \\
\hline $70-2,64-67$ & 69.02 & 0.48 & 10.74 & 2.79 & 1.44 & 0.036 & 1.95 & 0.68 & 1.60 & 1.89 & 0.065 & 7.22 & 0.0 & 1.79 & 0.67 & 100.37 \\
\hline $71-2,121-125$ & 71.95 & 0.38 & 8.33 & 2.55 & 0.85 & 0.023 & 1.48 & 0.48 & 1.25 & 1.48 & 0.054 & 9.36 & 0.0 & 1.80 & 1.15 & 101.13 \\
\hline $73-2,67-68$ & 71.12 & 0.47 & 10.50 & 2.80 & 1.13 & 0.030 & 1.72 & 0.88 & 1.53 & 1.70 & 0.051 & 7.33 & 0.0 & 0.81 & 0.91 & 100.98 \\
\hline $85-2.72-74$ & 67.83 & 0.49 & 11.16 & 2.91 & 1.33 & 0.032 & 1.68 & 1.37 & 1.50 & 1.72 & 0.067 & 6.84 & 0.0 & 2.58 & 0.88 & 100.39 \\
\hline \multicolumn{17}{|l|}{ lole 438B } \\
\hline $7-2,98-102$ & 67.84 & 0.50 & 12.74 & 2.83 & 1.64 & 0.038 & 1.68 & 1.42 & 1.65 & 1.93 & 0.078 & 6.69 & 0.0 & 1.54 & 0.38 & 100.95 \\
\hline $9-2,100-102$ & 63.52 & 0.62 & 13.73 & 3.43 & 1.72 & 0.071 & 1.68 & 2.76 & 2.50 & 1.80 & 0.093 & 5.10 & 1.46 & 0.30 & 0.32 & 99.09 \\
\hline $11-2,59-66$ & 65.15 & 0.62 & 13.73 & 3.34 & 1.72 & 0.055 & 1.65 & 3.12 & 1.73 & 1.50 & 0.110 & 5.96 & 0.0 & 1.43 & 0.40 & 100.52 \\
\hline $12-3,128-130$ & 68.64 & 0.51 & 11.01 & 2.67 & 1.77 & 0.040 & 1.51 & 1.97 & 1.55 & 1.63 & 0.083 & 8.09 & 0.0 & 0.32 & 0.40 & 100.20 \\
\hline $13-2,78-80$ & 67.59 & 0.47 & 9.85 & 2.46 & 1.58 & 0.035 & 1.63 & 0.39 & 1.30 & 1.44 & 0.073 & 8.66 & 3.70 & 0.32 & 0.58 & 100.07 \\
\hline $15-1,57-58$ & 65.80 & 0.60 & 12.65 & 2.98 & 2.03 & 0.049 & 2.12 & 1.71 & 1.75 & 1.84 & 0.093 & 8.21 & 0.0 & 0.35 & 0.38 & 100.56 \\
\hline $17-2,61-65$ & 64.89 & 0.65 & 14.99 & 3.08 & 2.27 & 0.042 & 2.90 & 0.97 & 1.96 & 2.48 & 0.110 & 6.30 & 0.0 & 0.16 & 0.16 & 100.96 \\
\hline $19-2,28-32$ & 66.53 & 0.52 & 12.06 & 4.80 & 2.46 & 0.031 & 2.99 & 0.96 & 1.97 & 2.74 & 0.087 & 4.94 & 0.0 & 0.31 & 0.09 & 100.49 \\
\hline $21-2,75-78$ & 67.44 & 0.52 & 13.67 & 2.81 & 1.55 & 0.024 & 1.89 & 2.01 & 2.29 & 2.10 & 0.068 & 3.95 & 0.0 & 0.56 & 0.11 & 98.99 \\
\hline $23-2,104-105$ & 64.83 & 0.62 & 14.43 & 2.47 & 2.15 & 0.032 & 2.38 & 0.91 & 2.08 & 2.39 & 0.084 & 5.26 & 0.0 & 1.34 & 0.12 & 99.09 \\
\hline
\end{tabular}

Site 439

Hole 439

\begin{tabular}{|c|c|c|c|c|c|c|c|c|c|c|c|c|c|c|c|c|}
\hline $7-2,90-93$ & 64.88 & 0.55 & 12.63 & 3.39 & 1.69 & 0.042 & 1.87 & 1.94 & 1.73 & 1.90 & 0.089 & 8.37 & 0.0 & 0.35 & 0.69 & 100.12 \\
\hline $9-2,16-19$ & 70.61 & 0.43 & 10.29 & 2.92 & 1.33 & 0.036 & 1.66 & 1.21 & 1.30 & 1.64 & 0.063 & 8.44 & 0.0 & 0.32 & 0.67 & 100.92 \\
\hline $11-2,29-32$ & 63.64 & 0.66 & 14.14 & 3.52 & 2.19 & 0.078 & 1.67 & 2.53 & 2.32 & 2.11 & 0.120 & 6.55 & 0.0 & 1.16 & 0.31 & 100.99 \\
\hline $13-1,86-88$ & 64.53 & 0.62 & 14.62 & 2.95 & 1.69 & 0.030 & 2.29 & 1.50 & 2.35 & 2.28 & 0.079 & 5.60 & 0.0 & 0.31 & 0.29 & 99.14 \\
\hline $15-2,105-107$ & 66.76 & 0.59 & 13.76 & 2.61 & 1.69 & 0.034 & 1.97 & 1.35 & 2.07 & 2.18 & 0.075 & 5.92 & 0.0 & 0.88 & 0.27 & 100.15 \\
\hline $17-3,28-31$ & 64.84 & 0.62 & 14.58 & 3.02 & 1.60 & 0.029 & 2.24 & 1.54 & 2.43 & 2.17 & 0.071 & 6.37 & 0.0 & 0.30 & 0.25 & 100.06 \\
\hline $19-1,57-58$ & 64.79 & 0.54 & 13.57 & 2.77 & 1.55 & 0.034 & 2.08 & 1.61 & 2.45 & 2.16 & 0.068 & 6.43 & 0.0 & 1.54 & 0.23 & 99.82 \\
\hline $21-2,68-70$ & 69.60 & 0.55 & 13.26 & 2.43 & 1.67 & 0.032 & 1.99 & 1.01 & 2.12 & 2.10 & 0.078 & 5.75 & 0.0 & 0.25 & 0.12 & 100.96 \\
\hline $23-2,17-19$ & 66.98 & 0.51 & 14.54 & 1.95 & 1.80 & 0.026 & 1.63 & $\begin{array}{l}1.01 \\
1.06\end{array}$ & 2.64 & 2.35 & 0.080 & 3.25 & 1.58 & 1.11 & 0.17 & 99.68 \\
\hline $29-1,114-119$ & 68.68 & 0.45 & 14.62 & 1.49 & 1.73 & 0.035 & 1.26 & 1.45 & 2.42 & 2.06 & 0.083 & 2.82 & 3.17 & 0.01 & 0.37 & 100.63 \\
\hline $31-2,86-88$ & 67.13 & 0.44 & 12.08 & 1.04 & 2.89 & 0.082 & 1.43 & 0.19 & 1.58 & 2.27 & 0.130 & 3.11 & 6.58 & 0.98 & 0.10 & 100.03 \\
\hline
\end{tabular}


TABLE 1 - Continued

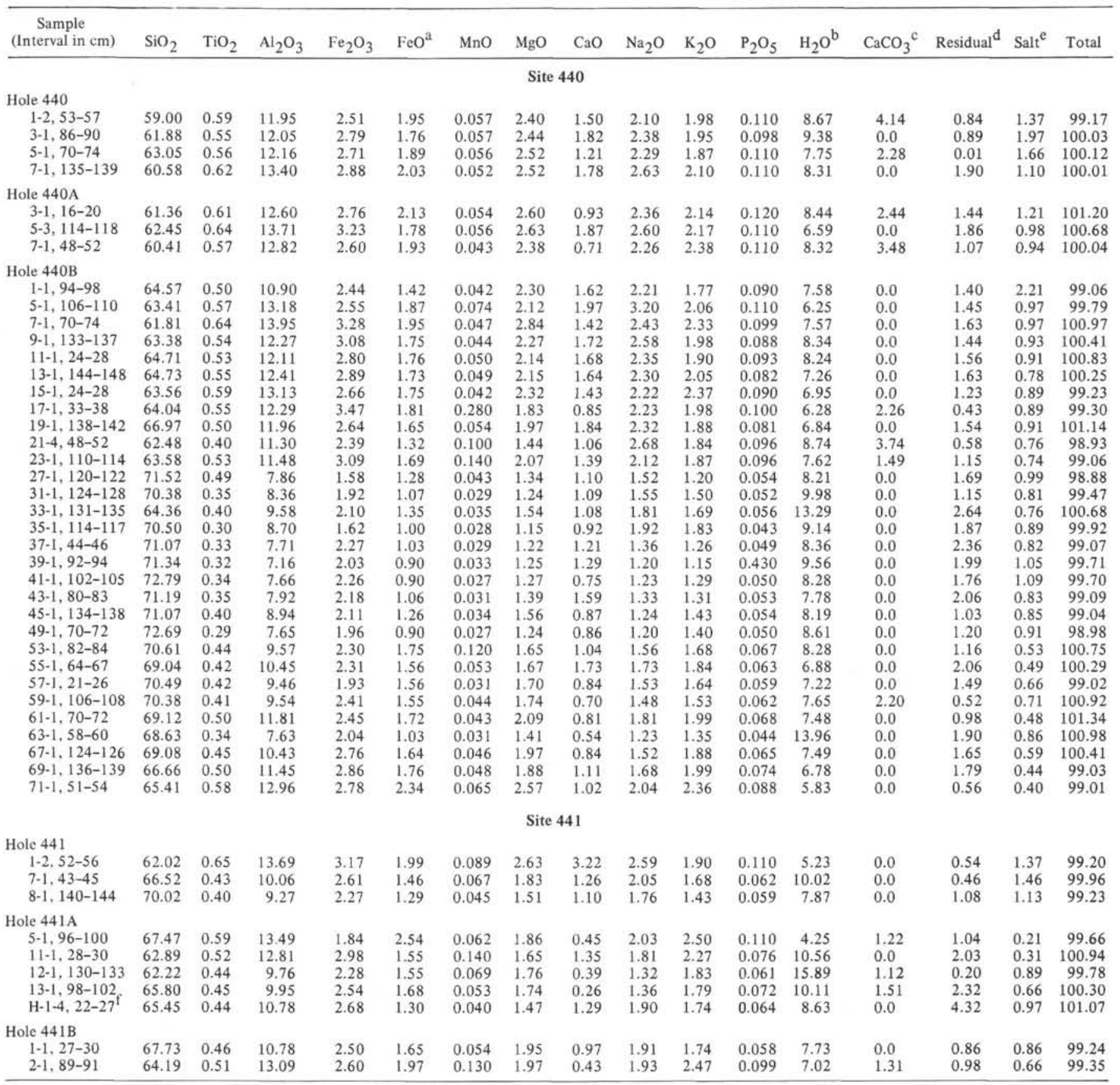

${ }^{\mathrm{a}} \mathrm{FeO}$ was determined colorimetrically with $o$-phenanthroline.

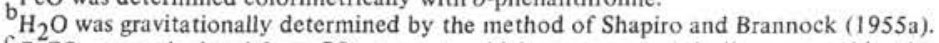

${ }^{\mathrm{c}} \mathrm{CaCO}_{3}$ was calculated from $\mathrm{CO}_{2}$ content, which was manometrically measured by the method of Shapiro and Brannock (1955b).

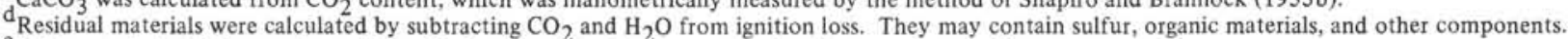

${ }_{\mathrm{e}}^{\mathrm{e}} \mathrm{Salt}$ was calculated from water soluble chlorine, assuming that pore water has the same composition as sea water.

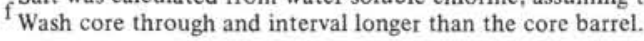


TABLE 2

Chemical Composition Recalculated by Excluding Carbonates, Residual Materials, Water, and Salts

\begin{tabular}{llllllllllllll}
\hline $\begin{array}{c}\text { Sample } \\
\text { (Interval in cm) }\end{array}$ & $\mathrm{SiO}_{2}$ & $\mathrm{TiO}_{2}$ & $\mathrm{Al}_{2} \mathrm{O}_{3}$ & $\mathrm{Fe}_{2} \mathrm{O}_{3}$ & $\mathrm{FeO}$ & $\mathrm{MnO}$ & $\mathrm{MgO}$ & $\mathrm{CaO}$ & $\mathrm{Na}_{2} \mathrm{O}$ & $\mathrm{K}_{2} \mathrm{O}$ & $\mathrm{P}_{2} \mathrm{O}_{5}$ & $\begin{array}{c}\mathrm{Fe}_{2} \mathrm{O}_{3} / \\
\mathrm{FeO}^{2}\end{array}$ & $\begin{array}{c}\mathrm{Total} \mathrm{Fe} \\
\text { as Fe }\end{array}$ \\
\hline
\end{tabular}

Site 434

Hole 434

\begin{tabular}{|c|c|c|c|c|c|c|c|c|c|c|c|c|c|}
\hline $1-1,140-142$ & 71.68 & 0.53 & 12.92 & 2.82 & 1.68 & 0.088 & 2.00 & 2.20 & 3.74 & 2.24 & 0.097 & 1.68 & 4.69 \\
\hline $1-4,80-82$ & 71.26 & 0.56 & 12.48 & 2.95 & 1.86 & 0.077 & 2.77 & 2.21 & 3.70 & 2.02 & 0.108 & 1.58 & 5.02 \\
\hline $2-1,100-104$ & 72.52 & 0.51 & 11.89 & 3.04 & 1.76 & 0.065 & 2.44 & 1.73 & 3.90 & 2.07 & 0.082 & 1.73 & 5.00 \\
\hline $4-1,80-84$ & 69.98 & 0.62 & 14.28 & 3.03 & 1.76 & 0.061 & 2.71 & 1.53 & 3.42 & 2.52 & 0.089 & 1.73 & 4.98 \\
\hline $5-1,120-124$ & 61.81 & 0.46 & 10.83 & 15.23 & 2.00 & 0.275 & 2.01 & 2.05 & 3.39 & 1.87 & 0.085 & 7.62 & 17.45 \\
\hline $6, \mathrm{CC}, 31-35$ & 75.19 & 0.46 & 11.17 & 3.53 & 1.51 & 0.059 & 1.83 & 1.19 & 3.05 & 1.95 & 0.070 & 2.34 & 5.20 \\
\hline $7-1,44-48$ & 77.09 & 0.46 & 10.96 & 2.72 & 1.36 & 0.044 & 1.94 & 1.17 & 2.33 & 1.86 & 0.058 & 1.99 & 4.23 \\
\hline $7-2,49-53$ & 76.99 & 0.45 & 10.72 & 2.61 & 1.55 & 0.057 & 2.10 & 1.11 & 2.53 & 1.83 & 0.062 & 1.68 & 4.33 \\
\hline $7-2,50-54$ & 66.45 & 0.66 & 15.42 & 3.61 & 1.92 & 0.063 & 3.24 & 1.69 & 4.14 & 2.72 & 0.093 & 1.88 & 5.75 \\
\hline $9-1,46-50$ & 74.97 & 0.47 & 11.24 & 3.01 & 1.29 & 0.066 & 2.24 & 1.45 & 3.23 & 1.98 & 0.060 & 2.33 & 4.44 \\
\hline $9-2,13-17$ & 78.20 & 0.41 & 9.78 & 2.96 & 1.15 & 0.036 & 2.17 & 0.98 & 2.53 & 1.72 & 0.057 & 2.58 & 4.24 \\
\hline $12-1,80-84$ & 76.20 & 0.47 & 11.13 & 2.73 & 1.88 & 0.546 & 1.37 & 1.20 & 2.43 & 1.98 & 0.079 & 1.45 & 4.81 \\
\hline $15-1,75-80$ & 69.60 & 0.63 & 14.13 & 3.35 & 1.83 & 0.058 & 2.59 & 1.98 & 3.30 & 2.45 & 0.091 & 1.83 & 5.38 \\
\hline $15-2,82-86$ & 69.15 & 0.60 & 14.66 & 3.39 & 1.69 & 0.061 & 2.19 & 2.95 & 3.23 & 1.98 & 0.083 & 2.00 & 5.28 \\
\hline $16-1,22-26$ & 75.06 & 0.49 & 11.56 & 2.97 & 1.36 & 0.052 & 1.95 & 1.43 & 3.14 & 1.91 & 0.068 & 2.19 & 4.48 \\
\hline $17-1,20-22$ & 75.92 & 0.49 & 11.27 & 2.73 & 1.43 & 0.044 & 2.15 & 1.17 & 2.74 & 1.98 & 0.063 & 1.91 & 4.33 \\
\hline $19-1,36-40$ & 66.79 & 0.41 & 10.20 & 13.52 & 1.79 & 0.246 & 2.39 & 0.11 & 2.73 & 1.71 & 0.092 & 7.54 & 15.51 \\
\hline $19-2,56-60$ & 63.23 & 0.43 & 9.97 & 16.70 & 1.66 & 0.316 & 2.47 & 0.49 & 2.96 & 1.64 & 0.158 & 10.09 & 18.54 \\
\hline $20-1,96-99$ & 77.51 & 0.48 & 11.09 & 2.87 & 1.43 & 0.059 & 2.03 & 0.44 & 2.20 & 1.82 & 0.069 & 2.01 & 4.46 \\
\hline $22-1,115-118$ & 76.14 & 0.48 & 11.62 & 3.08 & 1.22 & 0.061 & 1.86 & 1.23 & 2.35 & 1.90 & 0.056 & 2.52 & 4.44 \\
\hline $23-1,66-68$ & 76.92 & 0.46 & 10.96 & 2.92 & 1.29 & 0.048 & 1.85 & 1.10 & 2.53 & 1.87 & 0.058 & 2.26 & 4.35 \\
\hline $24-1,86-90$ & 74.07 & 0.47 & 10.84 & 4.73 & 1.52 & 0.130 & 2.56 & 0.95 & 2.59 & 1.98 & 0.154 & 3.12 & 6.42 \\
\hline $25-1,54-58$ & 73.85 & 0.48 & 11.72 & 4.83 & 1.69 & 0.124 & 2.22 & 0.14 & 2.88 & 1.96 & 0.097 & 2.86 & 6.71 \\
\hline $26-1,40-44$ & 73.92 & 0.53 & 12.41 & 3.27 & 1.53 & 0.056 & 2.16 & 1.25 & 2.74 & 2.07 & 0.075 & 2.14 & 4.96 \\
\hline $27-1,86-90$ & 63.48 & 0.31 & 10.45 & 16.41 & 1.69 & 0.310 & 2.71 & 0.09 & 2.59 & 1.84 & 0.126 & 9.73 & 18.29 \\
\hline $28-2,76-79$ & 72.65 & 0.58 & 13.25 & 4.05 & 1.74 & 0.283 & 1.69 & 1.13 & 2.28 & 2.24 & 0.120 & 2.33 & 5.98 \\
\hline $30-1,81-84$ & 78.00 & 0.46 & 10.65 & 2.45 & 1.44 & 0.064 & 1.67 & 1.11 & 2.33 & 1.74 & 0.072 & 1.70 & 4.06 \\
\hline $31-1,43-47$ & 74.30 & 0.54 & 12.84 & 2.59 & 1.94 & 0.172 & 1.92 & 0.79 & 2.73 & 2.10 & 0.087 & 1.33 & 4.74 \\
\hline $33-1,75-79$ & 67.58 & 0.48 & 11.96 & 9.32 & 1.91 & 0.219 & 2.46 & 1.25 & 2.80 & 1.90 & 0.109 & 4.88 & 11.44 \\
\hline $33-2,84-88$ & 72.19 & 0.50 & 12.51 & 4.32 & 1.74 & 0.120 & 2.05 & 1.08 & 3.39 & 2.01 & 0.086 & 2.48 & 6.25 \\
\hline Iole 434A & & & & & & & & & & & & & \\
\hline $1-1,56-60$ & 67.19 & 0.68 & 14.78 & 3.14 & 2.14 & 0.079 & 2.79 & 3.29 & 3.66 & 2.14 & 0.107 & 1.47 & 5.52 \\
\hline $1-5$ & 69.24 & 0.65 & 13.74 & 3.05 & 2.09 & 0.068 & 2.99 & 1.86 & 3.74 & 2.45 & 0.117 & 1.46 & 5.37 \\
\hline Iole 434B & & & & & & & & & & & & & \\
\hline $3-1,60-64$ & 71.62 & 0.57 & 13.92 & 3.50 & 1.60 & 0.073 & 1.98 & 1.64 & 2.57 & 2.42 & 0.098 & 2.19 & 5.28 \\
\hline $4-1,42-46$ & 73.48 & 0.58 & 12.97 & 3.44 & 1.54 & 0.051 & 1.84 & 1.48 & 2.45 & 2.09 & 0.077 & 2.23 & 5.16 \\
\hline $7-1,35-39$ & 76.96 & 0.51 & 11.28 & 2.88 & 1.60 & 0.273 & 1.59 & 0.81 & 2.14 & 1.90 & 0.081 & 1.79 & 4.66 \\
\hline $8-2,66-70$ & 77.48 & 0.51 & 10.43 & 3.43 & 1.84 & 0.143 & 1.79 & 0.22 & 2.34 & 1.74 & 0.086 & 1.86 & 5.47 \\
\hline $9-2,60-64$ & 83.47 & 0.35 & 7.82 & 1.54 & 1.40 & 0.050 & 1.31 & 0.91 & 1.73 & 1.36 & 0.055 & 1.10 & 3.11 \\
\hline $10-1,80-84$ & 76.78 & 0.52 & 11.99 & 2.50 & 1.81 & 0.212 & 1.60 & 0.40 & 2.34 & 1.76 & 0.081 & 1.38 & 4.52 \\
\hline $11-2,76-80$ & 74.17 & 0.49 & 11.55 & 4.49 & 2.14 & 0.217 & 1.70 & 0.74 & 2.30 & 2.09 & 0.114 & 2.10 & 6.87 \\
\hline $15-2,100-104$ & 77.88 & 0.46 & 11.13 & 2.45 & 1.61 & 0.044 & 1.42 & 1.06 & 2.04 & 1.84 & 0.066 & 1.52 & 4.23 \\
\hline $16-1,90-94$ & 78.54 & 0.37 & 10.75 & 1.91 & 1.30 & 0.038 & 1.23 & 1.35 & 2.66 & 1.78 & 0.064 & 1.47 & 3.36 \\
\hline $17-1,76-80$ & 75.08 & 0.49 & 11.69 & 3.46 & 1.65 & 0.131 & 2.04 & 0.87 & 2.42 & 2.08 & 0.095 & 2.10 & 5.30 \\
\hline $19-1,110-114$ & 71.15 & 0.59 & 14.66 & 3.66 & 1.60 & 0.117 & 2.04 & 1.10 & 2.18 & 2.83 & 0.084 & 2.30 & 5.44 \\
\hline $20-1,60-64$ & 73.81 & 0.52 & 13.42 & 2.36 & 1.65 & 0.045 & 1.80 & 1.47 & 2.67 & 2.19 & 0.078 & 1.43 & 4.19 \\
\hline $25-1,56-60$ & 77.24 & 0.40 & 11.83 & 2.00 & 1.36 & 0.071 & 0.99 & 1.56 & 2.70 & 1.78 & 0.058 & 1.47 & 3.51 \\
\hline $25-2,95-99$ & 74.02 & 0.52 & 13.69 & 2.97 & 1.86 & 0.142 & 1.68 & 0.16 & 2.36 & 2.51 & 0.080 & 1.59 & 5.04 \\
\hline $26-1,100-104$ & 71.92 & 0.55 & 13.78 & 3.88 & 1.73 & 0.161 & 2.03 & 0.92 & 2.41 & 2.55 & 0.087 & 2.25 & 5.80 \\
\hline $30-1,82-84$ & 73.11 & 0.55 & 14.02 & 3.51 & 1.57 & 0.043 & 1.97 & 0.66 & 2.13 & 2.38 & 0.065 & 2.24 & 5.25 \\
\hline $32-1,84-88$ & 73.49 & 0.55 & 13.40 & 3.37 & 1.73 & 0.325 & 1.72 & 0.48 & 2.36 & 2.47 & 0.102 & 1.94 & 5.30 \\
\hline $33-1,86-90$ & 73.36 & 0.55 & 13.45 & 3.23 & 1.67 & 0.107 & 1.90 & 0.66 & 2.45 & 2.53 & 0.082 & 1.93 & 5.09 \\
\hline $34-2,60-64$ & 73.92 & 0.51 & 13.44 & 2.51 & 1.86 & 0.106 & 1.74 & 0.87 & 2.44 & 2.40 & 0.204 & 1.35 & 4.57 \\
\hline $35-1,86-90$ & 73.81 & 0.56 & 12.93 & 3.15 & 1.88 & 0.092 & 2.15 & 0.91 & 2.47 & 1.97 & 0.081 & 1.67 & 5.24 \\
\hline $36-1,15-19$ & 78.02 & 0.45 & 10.87 & 2.58 & 1.44 & 0.037 & 1.75 & 0.95 & 2.09 & 1.75 & 0.059 & 1.79 & 4.19 \\
\hline \multicolumn{14}{|c|}{ Site 435} \\
\hline \multicolumn{14}{|l|}{ Hole 435} \\
\hline $1-3,40-44$ & 69.67 & 0.63 & 13.72 & 3.21 & 2.06 & 0.069 & 2.56 & 2.13 & 3.76 & 2.07 & 0.111 & 1.56 & 5.50 \\
\hline $2-2,50-54$ & 67.30 & 0.72 & 14.87 & 3.33 & 2.30 & 0.069 & 2.80 & 2.33 & 3.81 & 2.35 & 0.122 & 1.45 & 5.89 \\
\hline $4-2,40-44$ & 70.14 & 0.62 & 13.22 & 4.21 & 1.99 & 0.057 & 2.24 & 1.72 & 3.60 & 2.10 & 0.106 & 2.11 & 6.42 \\
\hline $5-3,28-32$ & 69.17 & 0.68 & 14.43 & 3.09 & 2.19 & 0.057 & 2.64 & 1.87 & 3.23 & 2.51 & 0.121 & 1.41 & 5.52 \\
\hline
\end{tabular}


TABLE 2 - Continued

\begin{tabular}{|c|c|c|c|c|c|c|c|c|c|c|c|c|c|}
\hline $\begin{array}{c}\text { Sample } \\
\text { (Interval in } \mathrm{cm} \text { ) }\end{array}$ & $\mathrm{SiO}_{2}$ & $\mathrm{TiO}_{2}$ & $\mathrm{Al}_{2} \mathrm{O}_{3}$ & $\mathrm{Fe}_{2} \mathrm{O}_{3}$ & $\mathrm{FeO}$ & $\mathrm{MnO}$ & $\mathrm{MgO}$ & $\mathrm{CaO}$ & $\mathrm{Na}_{2} \mathrm{O}$ & $\mathrm{K}_{2} \mathrm{O}$ & $\mathrm{P}_{2} \mathrm{O}_{5}$ & $\begin{array}{c}\mathrm{Fe}_{2} \mathrm{O}_{3} / \\
\mathrm{FeO}\end{array}$ & $\begin{array}{c}\text { Total Fe } \\
\text { as } \mathrm{Fe}_{2} \mathrm{O}_{3}\end{array}$ \\
\hline \multicolumn{14}{|c|}{ Site 435 (cont.) } \\
\hline \multicolumn{14}{|l|}{$\begin{array}{c}\text { Hole } 435 \text { (cont.) } \\
6-1,100-104\end{array}$} \\
\hline $6-1,100-104$ & 69.90 & 0.61 & 14.81 & 2.89 & 1.87 & 0.069 & 2.40 & 1.28 & 3.46 & 2.61 & 0.092 & 1.55 & 4.97 \\
\hline $7-3,100-104$ & 68.32 & 0.65 & 14.43 & 3.06 & 2.06 & 0.070 & 2.50 & 3.31 & 3.43 & 2.06 & 0.128 & 1.49 & 5.34 \\
\hline $8-2,70-73$ & 61.90 & 0.61 & 13.65 & 10.83 & 2.08 & 0.175 & 2.65 & 2.55 & 3.55 & 1.89 & 0.104 & 5.20 & 13.15 \\
\hline $9-2,70-73$ & 71.94 & 0.52 & 12.84 & 2.41 & 1.73 & 0.063 & 1.91 & 2.73 & 3.82 & 1.95 & 0.089 & 1.39 & 4.33 \\
\hline $13-3,80-84$ & 75.86 & 0.49 & 10.58 & 2.70 & 1.55 & 0.039 & 1.98 & 2.02 & 2.94 & 1.77 & 0.084 & 1.74 & 4.42 \\
\hline $14-1,14-18$ & 78.42 & 0.41 & 9.43 & 2.35 & 1.28 & 0.040 & 1.96 & 1.86 & 3.03 & 1.11 & 0.109 & 1.84 & 3.77 \\
\hline $15-2,10-14$ & 76.03 & 0.35 & 11.24 & 1.69 & 1.29 & 0.060 & 1.52 & 2.23 & 3.12 & 2.39 & 0.077 & 1.31 & 3.13 \\
\hline $16-1,72-74$ & 77.90 & 0.38 & 9.94 & 1.92 & 1.45 & 0.049 & 1.26 & 2.07 & 3.06 & 1.90 & 0.076 & 1.32 & 3.53 \\
\hline $29-1,59-63$ & 69.54 & 0.62 & 14.64 & 4.00 & 1.92 & 0.064 & 2.54 & 1.35 & 2.60 & 2.62 & 0.098 & 2.08 & 6.13 \\
\hline \multicolumn{14}{|l|}{ Hole 435A } \\
\hline $1-3,54-58$ & 66.79 & 0.70 & 15.12 & 3.32 & 2.30 & 0.071 & 2.77 & 2.81 & 3.74 & 2.25 & 0.120 & 1.44 & 5.88 \\
\hline $2-1,43-47$ & 81.24 & 0.35 & 7.55 & 1.99 & 1.13 & 0.032 & 1.46 & 2.10 & 2.79 & 1.28 & 0.070 & 1.75 & 3.25 \\
\hline $3-2,66-70$ & 82.75 & 0.32 & 7.46 & 1.66 & 1.08 & 0.053 & 1.30 & 1.34 & 2.70 & 1.27 & 0.066 & 1.54 & 2.86 \\
\hline $4-2,50-54$ & 79.38 & 0.42 & 9.21 & 2.07 & 1.47 & 0.045 & 1.88 & 1.31 & 2.61 & 1.55 & 0.061 & 1.40 & 3.70 \\
\hline $5-3,90-94$ & 76.45 & 0.46 & 10.81 & 2.47 & 1.50 & 0.046 & 1.76 & 1.90 & 2.78 & 1.73 & 0.083 & 1.65 & 4.14 \\
\hline $6-1,58-62$ & 78.99 & 0.42 & 9.53 & 2.78 & 1.27 & 0.039 & 1.44 & 1.42 & 2.54 & 1.50 & 0.078 & 2.18 & 4.20 \\
\hline $7-1,46-50$ & 79.20 & 0.42 & 9.33 & 2.26 & 1.44 & 0.033 & 1.84 & 1.29 & 2.59 & 1.52 & 0.071 & 1.58 & 3.86 \\
\hline $8-1,43-47$ & 85.31 & 0.30 & 5.95 & 1.54 & 0.98 & 0.023 & 1.34 & 1.13 & 2.39 & 0.98 & 0.064 & 1.57 & 2.63 \\
\hline $9-1,110-114$ & 80.61 & 0.36 & 8.81 & 2.03 & 1.15 & 0.029 & 1.59 & 1.12 & 2.69 & 1.56 & 0.054 & 1.76 & 3.31 \\
\hline $10-1,62-66$ & 77.87 & 0.44 & 10.01 & 2.72 & 1.28 & 0.048 & 2.03 & 1.47 & 2.43 & 1.62 & 0.073 & 2.13 & 4.14 \\
\hline $11-2,66-70$ & 80.23 & 0.39 & 8.98 & 2.46 & 1.20 & 0.053 & 1.66 & 1.03 & 2.39 & 1.50 & 0.108 & 2.04 & 3.80 \\
\hline \multicolumn{14}{|c|}{ Site 436} \\
\hline \multicolumn{14}{|l|}{ Hole 436} \\
\hline $1-1,50-54$ & 66.46 & 0.70 & 15.43 & 3.73 & 1.96 & 0.064 & 3.08 & 2.11 & 3.74 & 2.63 & 0.109 & 1.90 & 5.91 \\
\hline $1-4,50-54$ & 67.42 & 0.60 & 14.73 & 4.67 & 1.84 & 0.089 & 2.14 & 2.35 & 3.67 & 2.39 & 0.101 & 2.54 & 6.71 \\
\hline $2-1,110-114$ & 66.52 & 0.68 & 14.79 & 4.28 & 2.46 & 0.089 & 2.65 & 2.58 & 3.53 & 2.32 & 0.107 & 1.74 & 7.01 \\
\hline $3-3,100-104$ & 68.06 & 0.62 & 15.09 & 3.51 & 1.85 & 0.061 & 2.53 & 1.54 & 3.79 & 2.86 & 0.091 & 1.89 & 5.56 \\
\hline $4-2,130-134$ & 68.11 & 0.61 & 14.01 & 4.02 & 1.65 & 0.053 & 3.19 & 1.86 & 4.06 & 2.36 & 0.090 & 2.43 & 5.86 \\
\hline $4-4,130-134$ & 66.73 & 0.67 & 15.23 & 4.06 & 1.90 & 0.073 & 2.98 & 1.90 & 3.52 & 2.84 & 0.096 & 2.13 & 6.17 \\
\hline $5-2,14-18$ & 65.96 & 0.67 & 15.72 & 3.60 & 2.10 & 0.075 & 2.76 & 2.92 & 3.81 & 2.29 & 0.106 & 1.72 & 5.93 \\
\hline $5-4,14-18$ & 66.71 & 0.66 & 15.18 & 4.15 & 1.98 & 0.076 & 2.97 & 1.99 & 3.53 & 2.65 & 0.108 & 2.10 & 6.35 \\
\hline $6-3,80-84$ & 66.48 & 0.67 & 15.78 & 3.55 & 2.03 & 0.067 & 2.93 & 2.26 & 3.49 & 2.63 & 0.107 & 1.75 & 5.81 \\
\hline $6-3,84-88$ & 68.70 & 0.59 & 14.81 & 3.02 & 1.85 & 0.070 & 2.60 & 2.02 & 3.80 & 2.48 & 0.091 & 1.63 & 5.07 \\
\hline $7-6,6-10$ & 66.50 & 0.68 & 15.52 & 3.75 & 1.87 & 0.059 & 2.92 & 2.17 & 3.71 & 2.71 & 0.108 & 2.01 & 5.83 \\
\hline $8-2,50-54$ & 68.35 & 0.59 & 14.56 & 3.28 & 1.82 & 0.081 & 2.53 & 2.45 & 3.91 & 2.32 & 0.101 & 1.81 & 5.30 \\
\hline $8-4,50-54$ & 67.94 & 0.62 & 14.42 & 3.53 & 1.88 & 0.072 & 2.62 & 2.19 & 4.30 & 2.33 & 0.097 & 1.88 & 5.62 \\
\hline $9-3,113-118$ & 70.05 & 0.52 & 13.38 & 3.73 & 1.52 & 0.055 & 2.67 & 1.73 & 3.87 & 2.41 & 0.073 & 2.45 & 5.42 \\
\hline $9-6,41-46$ & 66.09 & 0.70 & 15.69 & 4.12 & 1.72 & 0.060 & 3.49 & 1.58 & 3.50 & 2.95 & 0.097 & 2.40 & 6.04 \\
\hline $10-2,113-118$ & 69.86 & 0.59 & 13.77 & 3.61 & 1.53 & 0.063 & 2.83 & 1.50 & 3.69 & 2.47 & 0.081 & 2.36 & 5.31 \\
\hline $10-5,113-118$ & 68.25 & 0.53 & 14.32 & 3.99 & 1.81 & 0.081 & 2.74 & 1.81 & 3.96 & 2.41 & 0.083 & 2.20 & 6.00 \\
\hline $11-2,86-90$ & 69.00 & 0.57 & 13.36 & 3.95 & 1.62 & 0.067 & 3.10 & 1.76 & 4.15 & 2.35 & 0.079 & 2.43 & 5.75 \\
\hline $11-5,86-90$ & 67.61 & 0.56 & 15.30 & 3.26 & 1.88 & 0.077 & 2.43 & 2.56 & 3.96 & 2.25 & 0.119 & 1.74 & 5.35 \\
\hline $12-2,36-40$ & 69.35 & 0.57 & 14.26 & 3.48 & 1.29 & 0.068 & 2.48 & 1.87 & 4.15 & 2.38 & 0.094 & 2.71 & 4.92 \\
\hline $12-4,36-40$ & 73.17 & 0.36 & 13.33 & 1.89 & 1.28 & 0.079 & 1.62 & 1.31 & 3.66 & 3.24 & 0.063 & 1.47 & 3.31 \\
\hline $13-2,73-75$ & 68.31 & 0.56 & 13.18 & 4.85 & 1.64 & 0.080 & 2.80 & 1.78 & 4.46 & 2.26 & 0.083 & 2.96 & 6.68 \\
\hline $13-4,73-75$ & 70.41 & 0.55 & 13.28 & 3.13 & 1.67 & 0.067 & 2.55 & 1.82 & 4.05 & 2.39 & 0.083 & 1.88 & 4.98 \\
\hline $14-1,60-64$ & 71.29 & 0.38 & 13.10 & 3.57 & 1.40 & 0.079 & 1.74 & 1.25 & 3.76 & 3.39 & 0.050 & 2.54 & 5.13 \\
\hline $14-3,60-64$ & 74.02 & 0.33 & 13.03 & 1.78 & 1.09 & 0.054 & 1.20 & 1.69 & 3.69 & 3.06 & 0.050 & 1.64 & 2.99 \\
\hline $15-1,90-94$ & 72.57 & 0.49 & 12.46 & 2.59 & 1.54 & 0.054 & 2.32 & 1.94 & 3.88 & 2.06 & 0.091 & 1.69 & 4.30 \\
\hline $15-6,30-34$ & 74.02 & 0.32 & 12.93 & 1.65 & 1.18 & 0.063 & 1.37 & 1.37 & 3.62 & 3.43 & 0.054 & 1.40 & 2.96 \\
\hline $16-2,90-94$ & 75.09 & 0.44 & 11.07 & 3.07 & 1.36 & 0.052 & 2.10 & 1.42 & 3.44 & 1.89 & 0.067 & 2.26 & 4.58 \\
\hline $17-4,30-34$ & 70.08 & 0.59 & 13.70 & 3.43 & 1.67 & 0.060 & 2.65 & 1.54 & 3.71 & 2.50 & 0.083 & 2.05 & 5.28 \\
\hline $18-2,34-38$ & 70.85 & 0.51 & 12.89 & 3.77 & 1.57 & 0.068 & 2.11 & 1.84 & 4.06 & 2.27 & 0.068 & 2.39 & 5.52 \\
\hline $19-1,66-70$ & 70.85 & 0.48 & 13.23 & 3.40 & 1.39 & 0.073 & 1.82 & 2.00 & 4.08 & 2.54 & 0.132 & 2.45 & 4.94 \\
\hline $19-4,86-90$ & 70.72 & 0.51 & 13.19 & 4.18 & 1.33 & 0.048 & 2.27 & 2.02 & 3.57 & 2.11 & 0.062 & 3.15 & 5.65 \\
\hline $20-1,105-109$ & 73.04 & 0.44 & 13.15 & 2.28 & 1.71 & 0.061 & 1.58 & 1.72 & 3.68 & 2.27 & 0.067 & 1.33 & 4.18 \\
\hline $21-2,64-68$ & 69.67 & 0.60 & 14.95 & 2.89 & 1.73 & 0.057 & 2.17 & 1.68 & 3.51 & 2.64 & 0.095 & 1.68 & 4.81 \\
\hline $22-1,52-56$ & 68.23 & 0.58 & 14.33 & 4.44 & 1.64 & 0.060 & 2.75 & 1.40 & 3.66 & 2.83 & 0.070 & 2.71 & 6.26 \\
\hline $23-2,35-39$ & 69.37 & 0.60 & 14.71 & 3.42 & 1.50 & 0.046 & 2.37 & 1.66 & 3.63 & 2.62 & 0.069 & 2.28 & 5.09 \\
\hline $23-5,35-39$ & 69.52 & 0.59 & 14.60 & 2.97 & 1.83 & 0.063 & 2.37 & 1.64 & 3.57 & 2.75 & 0.088 & 1.62 & 5.01 \\
\hline $24-1,53-57$ & 69.18 & 0.60 & 15.14 & 3.60 & 1.45 & 0.065 & 2.34 & 1.33 & 3.38 & 2.83 & 0.081 & 2.49 & 5.21 \\
\hline $25-1,74-78$ & 70.70 & 0.53 & 14.23 & 3.75 & 1.23 & 0.054 & 2.11 & 1.15 & 3.28 & 2.89 & 0.068 & 3.04 & 5.12 \\
\hline $27-1,106-110$ & 68.71 & 0.60 & 15.77 & 3.84 & 1.45 & 0.051 & 2.31 & 1.08 & 3.24 & 2.89 & 0.067 & 2.64 & 5.45 \\
\hline
\end{tabular}


TABLE 2 - Continued

\begin{tabular}{|c|c|c|c|c|c|c|c|c|c|c|c|c|c|}
\hline $\begin{array}{c}\text { Sample } \\
\text { (Interval in } \mathrm{cm} \text { ) }\end{array}$ & $\mathrm{SiO}_{2}$ & $\mathrm{TiO}_{2}$ & $\mathrm{Al}_{2} \mathrm{O}_{3}$ & $\mathrm{Fe}_{2} \mathrm{O}_{3}$ & $\mathrm{FeO}$ & $\mathrm{MnO}$ & $\mathrm{MgO}$ & $\mathrm{CaO}$ & $\mathrm{Na}_{2} \mathrm{O}$ & $\mathrm{K}_{2} \mathrm{O}$ & $\mathrm{P}_{2} \mathrm{O}_{5}$ & $\begin{array}{c}\mathrm{Fe}_{2} \mathrm{O}_{3} / \\
\mathrm{FeO}\end{array}$ & $\begin{array}{l}\text { Total Fe } \\
\text { as } \mathrm{Fe}_{2} \mathrm{O}_{3}\end{array}$ \\
\hline \multicolumn{14}{|c|}{ Site 436 (Cont.) } \\
\hline \multicolumn{14}{|l|}{ Hole 436 (cont.) } \\
\hline $27-2,106-110$ & 68.30 & 0.61 & 14.73 & 4.55 & 1.62 & 0.045 & 2.71 & 1.13 & 3.43 & 2.80 & 0.067 & 2.81 & 6.34 \\
\hline $28-1,56-60$ & 69.88 & 0.58 & 15.00 & 3.71 & 1.28 & 0.043 & 2.36 & 0.94 & 3.39 & 2.76 & 0.073 & 2.90 & 5.13 \\
\hline $29-1,70-74$ & 71.10 & 0.46 & 14.28 & 3.38 & 1.29 & 0.082 & 1.67 & 1.64 & 3.44 & 2.58 & 0.067 & 2.63 & 4.81 \\
\hline $29-2,70-74$ & 71.35 & 0.42 & 14.15 & 3.54 & 0.87 & 0.072 & 1.85 & 1.05 & 3.29 & 3.34 & 0.062 & 4.05 & 4.52 \\
\hline $30-1,74-78$ & 72.21 & 0.41 & 14.14 & 2.67 & 1.04 & 0.131 & 1.51 & 1.14 & 3.27 & 3.41 & 0.060 & 2.58 & 3.82 \\
\hline $30-3,74-78$ & 69.54 & 0.58 & 15.17 & 3.98 & 1.14 & 0.060 & 2.27 & 1.28 & 3.13 & 2.78 & 0.071 & 3.49 & 5.25 \\
\hline $31-2,40-44$ & 71.39 & 0.47 & 13.41 & 3.91 & 1.05 & 0.199 & 1.87 & 1.53 & 3.43 & 2.56 & 0.188 & 3.71 & 5.08 \\
\hline $31-4,40-44$ & 67.48 & 0.63 & 16.08 & 4.79 & 1.08 & 0.708 & 1.53 & 1.41 & 3.48 & 2.73 & 0.084 & 4.44 & 5.99 \\
\hline $32-2,46-50$ & 68.81 & 0.61 & 14.92 & 5.10 & 0.89 & 0.623 & 1.62 & 1.07 & 3.42 & 2.86 & 0.078 & 5.74 & 6.09 \\
\hline $33-1,52-56$ & 69.54 & 0.53 & 14.98 & 4.19 & 0.99 & 0.384 & 1.56 & 1.21 & 3.46 & 3.05 & 0.121 & 4.24 & 5.28 \\
\hline $33-4,52-56$ & 66.71 & 0.64 & 15.90 & 5.12 & 1.12 & 0.342 & 2.25 & 1.44 & 3.56 & 2.81 & 0.101 & 4.56 & 6.37 \\
\hline $34-2,60-64$ & 67.73 & 0.64 & 15.39 & 5.09 & 1.08 & 0.197 & 2.45 & 1.38 & 3.24 & 2.72 & 0.091 & 4.71 & 6.29 \\
\hline $34-6,60-64$ & 68.81 & 0.59 & 14.83 & 5.16 & 0.86 & 0.184 & 2.28 & 1.16 & 3.35 & 2.69 & 0.080 & 5.96 & 6.12 \\
\hline $35-1,60-64$ & 67.59 & 0.65 & 15.69 & 5.24 & 0.93 & 1.008 & 0.93 & 0.87 & 3.93 & 3.08 & 0.090 & 5.64 & 6.27 \\
\hline $35-4,60-64$ & 65.16 & 0.72 & 16.89 & 5.84 & 0.98 & 0.134 & 2.62 & 1.03 & 3.36 & 3.17 & 0.089 & 5.97 & 6.93 \\
\hline $36-2,50-54$ & 66.89 & 0.67 & 17.11 & 5.64 & 0.66 & 0.485 & 1.74 & 0.86 & 2.76 & 3.03 & 0.151 & 8.58 & 6.37 \\
\hline $36-6,50-54$ & 67.89 & 0.57 & 16.68 & 4.37 & 0.90 & 0.260 & 1.54 & 0.94 & 3.28 & 3.47 & 0.097 & 4.86 & 5.37 \\
\hline $37-2,70-74$ & 67.30 & 0.64 & 16.59 & 5.61 & 0.74 & 0.304 & 1.99 & 0.76 & 2.87 & 3.10 & 0.105 & 7.61 & 6.43 \\
\hline $37-6,70-74$ & 66.96 & 0.49 & 18.08 & 4.43 & 0.35 & 0.407 & 2.76 & 0.74 & 3.21 & 2.49 & 0.081 & 12.58 & 4.82 \\
\hline $38-2,66-70$ & 66.91 & 0.57 & 16.66 & 5.42 & 0.37 & 0.559 & 1.76 & 1.02 & 3.28 & 3.34 & 0.129 & 14.83 & 5.82 \\
\hline $39-1,60-64$ & 68.70 & 0.62 & 15.46 & 4.60 & 0.97 & 0.303 & 1.69 & 1.60 & 2.95 & 2.94 & 0.162 & 4.73 & 5.68 \\
\hline $39-6,60-64$ & 61.81 & 0.73 & 20.21 & 7.49 & 0.10 & 2.327 & 0.19 & 0.74 & 2.63 & 3.43 & 0.348 & 76.56 & 7.60 \\
\hline $40-3,50-54$ & 61.17 & 0.74 & 20.52 & 7.55 & 0.11 & 2.359 & 0.20 & 0.64 & 2.28 & 4.12 & 0.309 & 68.49 & 7.67 \\
\hline $40-4,50-54$ & 61.30 & 0.73 & 20.26 & 7.39 & 0.26 & 2.517 & 0.19 & 0.68 & 2.16 & 4.19 & 0.325 & 28.39 & 7.68 \\
\hline \multicolumn{14}{|c|}{ Site 438} \\
\hline \multicolumn{14}{|l|}{ Hole 438} \\
\hline $1-2,98-102$ & 70.90 & 0.47 & 14.15 & 2.66 & 1.94 & 0.075 & 1.70 & 2.54 & 3.32 & 2.17 & 0.083 & 1.37 & 4.81 \\
\hline $3-2,60-64$ & 68.66 & 0.67 & 13.95 & 3.37 & 2.28 & 0.059 & 2.65 & 3.27 & 2.54 & 2.41 & 0.117 & 1.48 & 5.91 \\
\hline $5-4,30-34$ & 66.25 & 0.59 & 13.60 & 5.83 & 1.87 & 0.095 & 2.81 & 3.63 & 3.08 & 2.15 & 0.099 & 3.13 & 7.90 \\
\hline $7-2,60-64$ & 73.45 & 0.53 & 12.16 & 2.61 & 1.93 & 0.066 & 2.13 & 2.49 & 2.66 & 1.87 & 0.103 & 1.35 & 4.75 \\
\hline $9-2,132-136$ & 74.71 & 0.48 & 12.25 & 2.42 & 1.62 & 0.050 & 1.85 & 2.04 & 2.44 & 2.04 & 0.092 & 1.50 & 4.22 \\
\hline $10-3,100-104$ & 71.36 & 0.58 & 12.86 & 2.96 & 2.13 & 0.071 & 2.29 & 3.23 & 2.61 & 1.79 & 0.111 & 1.39 & 5.33 \\
\hline $11-2,76-80$ & 72.45 & 0.58 & 12.11 & 3.32 & 1.95 & 0.062 & 1.97 & 2.97 & 2.63 & 1.86 & 0.102 & 1.70 & 5.48 \\
\hline \multicolumn{14}{|l|}{ Hole 438A } \\
\hline $1-2,66-70$ & 65.37 & 0.66 & 14.96 & 5.30 & 2.00 & 0.092 & 2.53 & 3.98 & 3.04 & 1.96 & 0.122 & 2.64 & 7.52 \\
\hline $3-2,72-76$ & 68.48 & 0.58 & 14.14 & 4.56 & 1.88 & 0.075 & 2.57 & 2.12 & 3.14 & 2.36 & 0.101 & 2.43 & 6.65 \\
\hline $5-2,145-147$ & 73.41 & 0.49 & 13.47 & 2.38 & 1.61 & 0.059 & 1.67 & 1.61 & 3.13 & 2.06 & 0.105 & 1.48 & 4.16 \\
\hline $7-2,41-42$ & 78.29 & 0.52 & 10.72 & 2.50 & 1.79 & 0.036 & 1.97 & 0.29 & 1.99 & 1.80 & 0.094 & 1.40 & 4.49 \\
\hline $9-1,22-24$ & 80.10 & 0.46 & 9.23 & 2.20 & 1.50 & 0.039 & 1.57 & 1.17 & 2.16 & 1.50 & 0.075 & 1.47 & 3.87 \\
\hline $11-2,84-88$ & 83.00 & 0.39 & 7.74 & 1.94 & 1.28 & 0.036 & 1.41 & 0.65 & 2.27 & 1.21 & 0.076 & 1.52 & 3.37 \\
\hline $13-2,9-13$ & 82.38 & 0.37 & 7.52 & 1.73 & 1.36 & 0.034 & 1.40 & 2.21 & 1.75 & 1.16 & 0.074 & 1.27 & 3.24 \\
\hline $17-2,62-64$ & 75.96 & 0.54 & 11.34 & 2.60 & 1.97 & 0.046 & 2.17 & 1.36 & 2.00 & 1.92 & 0.088 & 1.32 & 4.79 \\
\hline $19-2,60-64$ & 77.96 & 0.46 & 10.12 & 2.24 & 1.79 & 0.044 & 1.81 & 1.64 & 2.08 & 1.79 & 0.075 & 1.26 & 4.23 \\
\hline $21-2,40-44$ & 77.35 & 0.51 & 10.46 & 2.47 & 1.91 & 0.042 & 2.02 & 1.22 & 2.16 & 1.77 & 0.083 & 1.29 & 4.59 \\
\hline $23-1,34-38$ & 79.95 & 0.45 & 9.36 & 2.30 & 1.67 & 0.036 & 1.61 & 1.13 & 1.84 & 1.59 & 0.072 & 1.37 & 4.16 \\
\hline $24-2,20-22$ & 76.76 & 0.53 & 10.81 & 2.85 & 1.74 & 0.039 & 1.93 & 1.19 & 2.23 & 1.84 & 0.086 & 1.64 & 4.78 \\
\hline $25-2,120-124$ & 76.04 & 0.50 & 11.12 & 2.66 & 1.69 & 0.045 & 1.83 & 2.15 & 2.06 & 1.84 & 0.079 & 1.57 & 4.54 \\
\hline $27-2,50-54$ & 81.35 & 0.42 & 8.75 & 2.00 & 1.41 & 0.032 & 1.59 & 1.34 & 1.53 & 1.50 & 0.072 & 1.42 & 3.57 \\
\hline $29-2$, & 83.86 & 0.36 & 7.32 & 2.16 & 1.10 & 0.020 & 1.40 & 0.79 & 1.65 & 1.29 & 0.054 & 1.98 & 3.38 \\
\hline $31-2,40-44$ & 85.00 & 0.36 & 7.61 & 2.04 & 1.14 & 0.029 & 1.22 & -0.20 & 1.39 & 1.32 & 0.087 & 1.79 & 3.30 \\
\hline $33-2,36-46$ & 76.21 & 0.45 & 10.70 & 3.01 & 1.54 & 0.064 & 1.34 & 2.86 & 2.15 & 1.58 & 0.092 & 1.95 & 4.73 \\
\hline $35-2,10-14$ & 77.17 & 0.36 & 11.14 & 1.93 & 1.64 & 0.055 & 1.42 & 1.65 & 2.44 & 2.11 & 0.073 & 1.18 & 3.76 \\
\hline $39-2,100-102$ & 79.72 & 0.44 & 9.58 & 2.31 & 1.53 & 0.034 & 1.43 & 0.86 & 1.59 & 1.70 & 0.804 & 1.51 & 4.01 \\
\hline $41-2,121-123$ & 78.88 & 0.45 & 9.93 & 2.43 & 1.43 & 0.032 & 1.57 & 1.56 & 1.87 & 1.77 & 0.072 & 1.71 & 4.02 \\
\hline $43-2,132-136$ & 75.06 & 0.52 & 11.74 & 3.07 & 1.83 & 0.044 & 1.91 & 1.49 & 2.19 & 2.08 & 0.074 & 1.68 & 5.10 \\
\hline $47-2,72-76$ & 77.25 & 0.48 & 10.26 & 2.32 & 1.77 & 0.041 & 2.00 & 0.96 & 1.77 & 1.85 & 1.294 & 1.31 & 4.29 \\
\hline $49-2,80-82$ & 77.38 & 0.51 & 10.71 & 2.39 & 1.93 & 0.044 & 2.10 & 1.11 & 1.81 & 1.93 & 0.083 & 1.24 & 4.54 \\
\hline $51-2,27-29$ & 72.05 & 0.60 & 13.15 & 2.72 & 2.27 & $\begin{array}{l}0.044 \\
0.049\end{array}$ & 2.53 & $\begin{array}{l}1.11 \\
1.77\end{array}$ & $\begin{array}{l}1.01 \\
2.35\end{array}$ & 2.43 & 0.089 & $\begin{array}{l}1.24 \\
1.19\end{array}$ & 5.24 \\
\hline $53-1,116-120$ & 77.98 & 0.49 & 10.01 & 2.46 & 1.75 & 0.032 & 2.07 & 1.19 & 2.07 & 1.87 & 0.075 & 1.41 & 4.41 \\
\hline $55-2,30-32$ & 79.49 & 0.47 & 9.64 & 2.29 & 1.63 & 0.032 & 1.78 & 0.83 & 1.98 & 1.79 & 0.072 & 1.40 & 4.10 \\
\hline $57-2,20-24$ & 76.29 & 0.51 & 11.04 & 2.54 & 1.88 & 0.038 & 2.23 & 1.12 & 2.24 & 2.02 & 0.080 & 1.35 & 4.63 \\
\hline $59-2,74-78$ & 75.32 & 0.50 & 11.02 & 2.89 & 1.77 & 0.033 & 2.32 & 1.83 & 2.09 & 2.15 & 0.080 & 1.63 & 4.85 \\
\hline $63-1,42-44$ & 80.74 & 0.45 & 9.23 & 2.22 & 1.51 & 0.048 & 1.79 & 0.77 & 1.55 & 1.62 & 0.072 & 1.47 & 3.90 \\
\hline
\end{tabular}


TABLE 2 - Continued

\begin{tabular}{|c|c|c|c|c|c|c|c|c|c|c|c|c|c|}
\hline $\begin{array}{c}\text { Sample } \\
\text { (Interval in } \mathrm{cm} \text { ) }\end{array}$ & $\mathrm{SiO}_{2}$ & $\mathrm{TiO}_{2}$ & $\mathrm{Al}_{2} \mathrm{O}_{3}$ & $\mathrm{Fe}_{2} \mathrm{O}_{3}$ & $\mathrm{FeO}$ & $\mathrm{MnO}$ & $\mathrm{MgO}$ & $\mathrm{CaO}$ & $\mathrm{Na}_{2} \mathrm{O}$ & $\mathrm{K}_{2} \mathrm{O}$ & $\mathrm{P}_{2} \mathrm{O}_{5}$ & $\begin{array}{c}\mathrm{Fe}_{2} \mathrm{O}_{3} / \\
\mathrm{FeO}\end{array}$ & $\begin{array}{l}\text { Total } \mathrm{Fe} \\
\text { as } \mathrm{Fe}_{2} \mathrm{O}\end{array}$ \\
\hline \multicolumn{14}{|c|}{ Site 438 (cont.) } \\
\hline Hole $438 \mathrm{~A}$ (cont.) & & & & & & & & & & & & & \\
\hline $65-1,77-79$ & 80.67 & 0.42 & 9.18 & 2.34 & 1.34 & 0.027 & 1.74 & 0.76 & 1.67 & 1.80 & 0.055 & 1.75 & 3.83 \\
\hline $70-2,64-67$ & 76.11 & 0.53 & 11.84 & 3.08 & 1.59 & 0.040 & 2.15 & 0.75 & 1.76 & 2.08 & 0.072 & 1.94 & 4.84 \\
\hline $71-2,121-125$ & 81.01 & 0.43 & 9.38 & 2.87 & 0.96 & 0.026 & 1.66 & 0.54 & 1.40 & 1.67 & 0.061 & 2.99 & 3.93 \\
\hline $73-2,67-68$ & 77.36 & 0.51 & 11.42 & 3.05 & 1.23 & 0.033 & 1.87 & 0.96 & 1.66 & 1.85 & 0.055 & 2.48 & 4.42 \\
\hline $85-2,72-74$ & 75.29 & 0.54 & 12.39 & 3.23 & 1.48 & 0.036 & 1.87 & 1.52 & 1.67 & 1.91 & 0.074 & 2.19 & 4.87 \\
\hline \multicolumn{14}{|l|}{ Hole 438B } \\
\hline $7-2,98-102$ & 73.47 & 0.54 & 13.80 & 3.06 & 1.78 & 0.041 & 1.81 & 1.54 & 1.78 & 2.09 & 0.084 & 1.72 & 5.04 \\
\hline $9-2,100-102$ & 69.10 & 0.67 & 14.94 & 3.73 & 1.87 & 0.077 & 1.83 & 3.00 & 2.71 & 1.96 & 0.101 & 1.99 & 5.81 \\
\hline $11-2,59-66$ & 70.26 & 0.67 & 14.81 & 3.60 & 1.85 & 0.059 & 1.78 & 3.36 & 1.87 & 1.62 & 0.119 & 1.94 & 5.66 \\
\hline $12-3,128-130$ & 75.11 & 0.56 & 12.05 & 2.92 & 1.94 & 0.044 & 1.66 & 2.16 & 1.70 & 1.78 & 0.091 & 1.51 & 5.08 \\
\hline $13-2,78-80$ & 77.86 & 0.54 & 11.35 & 2.84 & 1.82 & 0.040 & 1.88 & 0.44 & 1.50 & 1.66 & 0.084 & 1.56 & 4.86 \\
\hline $15-1,57-58$ & 71.82 & 0.65 & 13.81 & 3.26 & 2.22 & 0.053 & 2.31 & 1.87 & 1.91 & 2.01 & 0.102 & 1.47 & 5.72 \\
\hline $17-2,61-65$ & 68.79 & 0.69 & 15.89 & 3.26 & 2.41 & 0.045 & 3.07 & 1.03 & 2.08 & 2.63 & 0.117 & 1.36 & 5.94 \\
\hline $19-2,28-32$ & 69.92 & 0.55 & 12.67 & 5.04 & 2.59 & 0.033 & 3.14 & 1.01 & 2.07 & 2.88 & 0.091 & 1.95 & 7.91 \\
\hline $21-2,75-78$ & 71.46 & 0.55 & 14.48 & 2.97 & 1.64 & 0.025 & 2.00 & 2.13 & 2.43 & 2.23 & 0.072 & 1.81 & 4.80 \\
\hline $23-2,104-105$ & 70.18 & 0.67 & 15.62 & 2.67 & 2.33 & 0.035 & 2.58 & 0.99 & 2.25 & 2.59 & 0.091 & 1.15 & 5.26 \\
\hline
\end{tabular}

Hole 439

$\begin{array}{llll}7-2,90-93 & 71.52 & 0.61 & 13.92 \\ 9-2,16-19 & 77.18 & 0.47 & 11.25 \\ 11-2,29-32 & 68.45 & 0.71 & 15.21 \\ 13-1,86-88 & 69.43 & 0.67 & 15.73 \\ 15-2,105-107 & 71.72 & 0.63 & 14.78 \\ 17-3,28-31 & 69.62 & 0.67 & 15.65 \\ 19-1,57-58 & 70.72 & 0.59 & 14.81 \\ 21-2,68-70 & 73.39 & 0.58 & 13.98 \\ 23-2,17-19 & 71.58 & 0.55 & 15.54 \\ 29-1,114-119 & 72.85 & 0.48 & 15.51 \\ 31-2,86-88 & 75.21 & 0.49 & 13.53\end{array}$

Site 439

$\begin{array}{llllllllll}3.74 & 1.86 & 0.046 & 2.06 & 2.14 & 1.91 & 2.09 & 0.098 & 2.01 & 5.81 \\ 3.19 & 1.45 & 0.039 & 1.81 & 1.32 & 1.42 & 1.79 & 0.069 & 2.20 & 4.81 \\ 3.78 & 2.36 & 0.084 & 1.80 & 2.72 & 2.50 & 2.27 & 0.129 & 1.61 & 6.40 \\ 3.18 & 1.82 & 0.032 & 2.47 & 1.61 & 2.53 & 2.45 & 0.085 & 1.75 & 5.20 \\ 2.81 & 1.82 & 0.037 & 2.11 & 1.45 & 2.22 & 2.34 & 0.081 & 1.55 & 4.82 \\ 3.24 & 1.72 & 0.031 & 2.40 & 1.65 & 2.61 & 2.33 & 0.076 & 1.89 & 5.15 \\ 3.02 & 1.69 & 0.037 & 2.27 & 1.76 & 2.67 & 2.36 & 0.074 & 1.79 & 4.90 \\ 2.57 & 1.76 & 0.034 & 2.10 & 1.06 & 2.23 & 2.21 & 0.082 & 1.46 & 4.52 \\ 2.08 & 1.92 & 0.028 & 1.75 & 1.14 & 2.82 & 2.51 & 0.085 & 1.08 & 4.22 \\ 1.58 & 1.84 & 0.037 & 1.33 & 1.54 & 2.56 & 2.19 & 0.088 & 0.86 & 3.62 \\ 1.16 & 3.24 & 0.092 & 1.60 & 0.21 & 1.77 & 2.54 & 0.146 & 0.36 & 4.76\end{array}$

Hole 440

\begin{tabular}{|c|c|c|c|c|c|c|c|c|c|c|c|c|c|}
\hline $1-2,53-57$ & 70.12 & 0.70 & 14.20 & 2.99 & 2.32 & 0.068 & 2.85 & 1.78 & 2.50 & 2.35 & 0.131 & 1.29 & 5.56 \\
\hline $3-1,86-90$ & 70.49 & 0.63 & 13.73 & 3.18 & 2.00 & 0.065 & 2.78 & 2.07 & 2.72 & 2.22 & 0.112 & 1.59 & 5.41 \\
\hline $5-1,70-74$ & 71.30 & 0.63 & 13.75 & 3.06 & 2.14 & 0.063 & 2.85 & 1.37 & 2.59 & 2.11 & 0.124 & 1.43 & 5.44 \\
\hline $7-1,135-139$ & 68.30 & 0.70 & 15.11 & 3.25 & 2.29 & 0.059 & 2.84 & 2.01 & 2.96 & 2.37 & 0.124 & 1.42 & 5.79 \\
\hline \multirow{4}{*}{$\begin{array}{l}\text { le } 440 \mathrm{~A} \\
3-1,16-20 \\
5-3,114-118 \\
7-1,48-52\end{array}$} & & & & & & & & & & & & & \\
\hline & 69.99 & 0.70 & 14.37 & 3.15 & 2.43 & 0.062 & 2.97 & 1.06 & 2.69 & 2.44 & 0.137 & 1.30 & 5.85 \\
\hline & 68.44 & 0.70 & 15.02 & 3.54 & 1.95 & 0.061 & 2.88 & 2.05 & 2.85 & 2.38 & 0.121 & 1.82 & 5.71 \\
\hline & 70.06 & 0.66 & 14.87 & 3.02 & 2.24 & 0.050 & 2.77 & 0.82 & 2.62 & 2.76 & 0.128 & 1.35 & 5.51 \\
\hline \multicolumn{14}{|l|}{ le $440 \mathrm{~B}$} \\
\hline $1-1,94-98$ & 73.49 & 0.57 & 12.41 & 2.78 & 1.62 & 0.048 & 2.62 & 1.84 & 2.52 & 2.01 & 0.102 & 1.72 & 4.58 \\
\hline $5-1,106-110$ & 69.59 & 0.63 & 14.47 & 2.80 & 2.05 & 0.081 & 2.33 & 2.16 & 3.51 & 2.26 & 0.121 & 1.36 & 5.08 \\
\hline $7-1,70-74$ & 68.07 & 0.70 & 15.36 & 3.62 & 2.15 & 0.052 & 3.13 & 1.56 & 2.67 & 2.57 & 0.109 & 1.68 & 6.00 \\
\hline $9-1,133-137$ & 70.66 & 0.60 & 13.68 & 3.43 & 1.95 & 0.049 & 2.53 & 1.92 & 2.88 & 2.21 & 0.098 & 1.76 & 5.60 \\
\hline $11-1,24-28$ & 71.80 & 0.59 & 13.44 & 3.11 & 1.95 & 0.055 & 2.37 & 1.86 & 2.60 & 2.11 & 0.103 & 1.59 & 5.28 \\
\hline $13-1,144-148$ & 71.46 & 0.61 & 13.70 & 3.19 & 1.91 & 0.054 & 2.37 & 1.81 & 2.54 & 2.26 & 0.091 & 1.67 & 5.31 \\
\hline $15-1,24-28$ & 70.50 & 0.65 & 14.56 & 2.94 & 1.94 & 0.047 & 2.57 & 1.59 & 2.47 & 2.63 & 0.100 & 1.52 & 5.10 \\
\hline $17-1,33-38$ & 71.61 & 0.61 & 13.74 & 3.88 & 2.02 & 0.313 & 2.05 & 0.95 & 2.50 & 2.21 & 0.112 & 1.92 & 6.13 \\
\hline $19-1,138-142$ & 72.91 & 0.54 & 13.02 & 2.87 & 1.80 & 0.059 & 2.14 & 2.00 & 2.52 & 2.05 & 0.088 & 1.60 & 4.87 \\
\hline $21-4,48-52$ & 73.41 & 0.47 & 13.28 & 2.81 & 1.55 & 0.117 & 1.69 & 1.25 & 3.14 & 2.16 & 0.113 & 1.81 & 4.54 \\
\hline $23-1,110-114$ & 72.20 & 0.60 & 13.04 & 3.51 & 1.92 & 0.159 & 2.35 & 1.58 & 2.41 & 2.12 & 0.109 & 1.83 & 5.64 \\
\hline $27-1,120-122$ & 81.28 & 0.56 & 8.93 & 1.79 & 1.45 & 0.049 & 1.52 & 1.25 & 1.73 & 1.36 & 0.061 & 1.23 & 3.41 \\
\hline $31-1,124-128$ & 80.40 & 0.40 & 9.55 & 2.19 & 1.22 & 0.033 & 1.41 & 1.25 & 1.77 & 1.71 & 0.059 & 1.80 & 3.55 \\
\hline $33-1,131-135$ & 76.62 & 0.48 & 11.41 & 2.50 & 1.61 & 0.042 & 1.83 & 1.29 & 2.15 & 2.01 & 0.067 & 1.56 & 4.29 \\
\hline $35-1,114-117$ & 80.10 & 0.34 & 9.89 & 1.84 & 1.14 & 0.032 & 1.31 & 1.05 & 2.19 & 2.08 & 0.049 & 1.62 & 3.10 \\
\hline $37-1,44-46$ & 81.20 & 0.38 & 8.81 & 2.59 & 1.18 & 0.033 & 1.39 & 1.38 & 1.55 & 1.44 & 0.056 & 2.20 & 3.90 \\
\hline $39-1,92-94$ & 81.90 & 0.37 & 8.22 & 2.33 & 1.03 & 0.038 & 1.44 & 1.48 & 1.37 & 1.32 & 0.494 & 2.26 & 3.48 \\
\hline $41-1,102-105$ & 82.18 & 0.38 & 8.65 & 2.55 & 1.02 & 0.030 & 1.44 & 0.85 & 1.39 & 1.46 & 0.056 & 2.51 & 3.68 \\
\hline
\end{tabular}


TABLE 2 - Continued

\begin{tabular}{|c|c|c|c|c|c|c|c|c|c|c|c|c|c|}
\hline $\begin{array}{c}\text { Sample } \\
\text { (Interval in } \mathrm{cm} \text { ) }\end{array}$ & $\mathrm{SiO}_{2}$ & $\mathrm{TiO}_{2}$ & $\mathrm{Al}_{2} \mathrm{O}_{3}$ & $\mathrm{Fe}_{2} \mathrm{O}_{3}$ & $\mathrm{FeO}$ & $\mathrm{MnO}$ & $\mathrm{MgO}$ & $\mathrm{CaO}$ & $\mathrm{Na}_{2} \mathrm{O}$ & $\mathrm{K}_{2} \mathrm{O}$ & $\mathrm{P}_{2} \mathrm{O}_{5}$ & $\begin{array}{c}\mathrm{Fe}_{2} \mathrm{O}_{3} / \\
\mathrm{FeO}\end{array}$ & $\begin{array}{l}\text { Total Fe } \\
\text { as } \mathrm{Fe}_{2} \mathrm{O}_{3}\end{array}$ \\
\hline \multicolumn{14}{|c|}{ Site 440 (cont.) } \\
\hline \multicolumn{14}{|l|}{ Hole $440 \mathrm{~B}$ (cont.) } \\
\hline $43-1,80-83$ & 80.52 & 0.40 & 8.96 & 2.47 & 1.20 & 0.035 & 1.58 & 1.80 & 1.50 & 1.48 & 0.060 & 2.06 & 3.80 \\
\hline $45-1,134-138$ & 79.88 & 0.45 & 10.05 & 2.37 & 1.42 & 0.038 & 1.76 & 0.98 & 1.40 & 1.61 & 0.061 & 1.67 & 3.94 \\
\hline $49-1,70-72$ & 82.36 & 0.33 & 8.67 & 2.22 & 1.02 & 0.031 & 1.40 & 0.97 & 1.35 & 1.59 & 0.057 & 2.18 & 3.35 \\
\hline $53-1,82-84$ & 77.73 & 0.49 & 11.18 & 2.73 & 1.67 & 0.065 & 1.69 & 0.87 & 1.64 & 1.83 & 0.109 & 1.63 & 4.59 \\
\hline $55-1,64-67$ & 75.99 & 0.46 & 11.50 & 2.54 & 1.72 & 0.058 & 1.83 & 1.90 & 1.90 & 2.03 & 0.069 & 1.48 & 4.45 \\
\hline $57-1,21-26$ & 78.63 & 0.47 & 10.55 & 2.15 & 1.74 & 0.035 & 1.90 & 0.94 & 1.70 & 1.83 & 0.066 & 1.23 & 4.08 \\
\hline $59-1,106-108$ & 78.35 & 0.46 & 10.62 & 2.68 & 1.73 & 0.049 & 1.93 & 0.78 & 1.64 & 1.70 & 0.069 & 1.55 & 4.60 \\
\hline $61-1,70-72$ & 74.80 & 0.54 & 12.78 & 2.65 & 1.86 & 0.047 & 2.26 & 0.88 & 1.96 & 2.15 & 0.074 & 1.42 & 4.72 \\
\hline $63-1,58-60$ & 81.44 & 0.40 & 9.05 & 2.42 & 1.22 & 0.037 & 1.68 & 0.64 & 1.45 & 1.60 & 0.052 & 1.98 & 3.77 \\
\hline $67-1,124-126$ & 76.18 & 0.50 & 11.50 & 3.04 & 1.81 & 0.051 & 2.17 & 0.93 & 1.68 & 2.07 & 0.072 & 1.68 & 5.05 \\
\hline $69-1,136-139$ & 74.05 & 0.56 & 12.72 & 3.18 & 1.96 & 0.053 & 2.09 & 1.23 & 1.87 & 2.21 & 0.082 & 1.63 & 5.35 \\
\hline $71-1,51-54$ & 70.93 & 0.63 & 14.05 & 3.01 & 2.54 & 0.070 & 2.79 & 1.11 & 2.22 & 2.56 & 0.095 & 1.19 & 5.83 \\
\hline \multicolumn{14}{|c|}{ Site 441} \\
\hline Hole 441 & & & & & & & & & & & & & \\
\hline $1-2,52-56$ & 67.37 & 0.71 & 14.87 & 3.44 & 2.16 & 0.097 & 2.85 & 3.50 & 2.81 & 2.06 & 0.119 & 1.59 & 5.84 \\
\hline $7-1,43-45$ & 75.57 & 0.49 & 11.43 & 2.96 & 1.66 & 0.076 & 2.08 & 1.43 & 2.33 & 1.91 & 0.070 & 1.79 & 4.81 \\
\hline $8-1,140-144$ & 78.54 & 0.45 & 10.40 & 2.54 & 1.45 & 0.050 & 1.69 & 1.23 & 1.98 & 1.60 & 0.066 & 1.76 & 4.15 \\
\hline \multicolumn{14}{|l|}{ Hole 441A } \\
\hline $5-1,96-100$ & 72.60 & 0.63 & 14.51 & 1.98 & 2.73 & 0.067 & 2.00 & 0.48 & 2.19 & 2.69 & 0.118 & 0.72 & 5.01 \\
\hline $11-1,28-30$ & 71.43 & 0.59 & 14.55 & 3.38 & 1.76 & 0.159 & 1.88 & 1.53 & 2.06 & 2.58 & 0.086 & 1.92 & 5.34 \\
\hline $12-1,130-133$ & 76.17 & 0.54 & 11.95 & 2.79 & 1.90 & 0.084 & 2.15 & 0.48 & 1.62 & 2.24 & 0.075 & 1.47 & 4.90 \\
\hline $13-1,98-102$ & 76.78 & 0.53 & 11.61 & 2.97 & 1.96 & 0.062 & 2.03 & 0.31 & 1.58 & 2.09 & 0.084 & 1.51 & 5.15 \\
\hline $\mathrm{H}-1-4,22-27^{\mathrm{a}}$ & 75.10 & 0.50 & 12.37 & 3.07 & 1.49 & 0.046 & 1.69 & 1.48 & 2.18 & 2.00 & 0.073 & 2.06 & 4.73 \\
\hline \multicolumn{14}{|l|}{ Hole 441B } \\
\hline $1-1,27-30$ & 75.43 & 0.51 & 12.00 & 2.78 & 1.84 & 0.060 & 2.17 & 1.08 & 2.12 & 1.94 & 0.065 & 1.51 & 4.82 \\
\hline $2-1,89-91$ & 71.82 & 0.57 & 14.65 & 2.91 & 2.20 & 0.145 & 2.20 & 0.48 & 2.15 & 2.76 & 0.111 & 1.32 & 5.36 \\
\hline
\end{tabular}

${ }^{a}$ See Table 1, footnote f.

chapters) at the location nearest to samples selected for chemical analysis were obtained from the core description and plotted against the $\mathrm{SiO}_{2}$ content (Figure 3). The correlation is rather obscure considered over an entire core. If the core is divided into several parts, the correlation between $\mathrm{SiO}_{2}$ and siliceous organism content becomes clear within each part. This is noteworthy if we remember that the comparison of chemical analyses with microscopic observation was not done within the same sample and that the accuracy of microscopic determinations is usually lower than that of chemical analyses for silica. The correlation strongly suggests that the siliceous organisms are responsible for the higher $\mathrm{SiO}_{2}$ of DSDP samples in the Japan Trench. Furthermore, in comparison with the upper part of a core, the lower part at any given level of siliceous organism content tends to be higher in silica; namely, the deeper the part in the core, the steeper the correlation line (Figure 3). The dissolution-redeposition mechanism of silica during the process of the diagenetic maturation of siliceous ooze seems to explain the different slopes of the regression lines. According to the experiments conducted by hydrothermal conditions at $150^{\circ} \mathrm{C}$ and at room temperature (Kastner et al., 1977), siliceous tests of radiolarians or diatoms were corroded and silica as opal-CT lepispheres redeposited around them. The rate of diagenesis of siliceous ooze is strongly affected by temperature and time. The degree of diagenesis of marine sediments may be greater at depth. As diagenesis proceeds, the tests of siliceous organisms tend to disappear and the silica from the tests to be redeposited in the sediments. In the process, the total amount of silica in the sediments remains constant. As a result, the regression line on the $\mathrm{SiO}_{2}$ organisms diagram becomes steeper. Some samples (illustrated by closed circles in Figure $3 \mathrm{~A}$ ) show higher $\mathrm{SiO}_{2}$ content in spite of their lower siliceous organism content. These samples contain a great deal of volcanic glass, which may be responsible for the higher silica content.

Figure 4 shows the vertical variation of $\mathrm{SiO}_{2}$ at each site. $\mathrm{SiO}_{2}$ tends to be higher prior to the late Tertiary. Various types of diatomaceous deposits occur in various horizons of the Neogene strata in such Circum-Japan Sea areas as the Noto Peninsula. Upper Tertiary sediments of the central Japan Sea contain large diatom deposits (Sugisaki, 1979). From the evidence, we can conclude that the favorable environment for diatom development had prevailed in the Japan Trench as well as in the Japan Sea during the late Cenozoic.

\section{PRECIPITATION OF IRON AND MANGANESE}

Figure 5 shows the relationship between $\mathrm{Fe}_{2} \mathrm{O}_{3}$ and total iron as $\mathrm{Fe}_{2} \mathrm{O}_{3}$ in samples from Site 438. The slope of the regression line is nearly 1 . The regression lines for other sites also have a slope of approximately 1, except for Site 440 (Table 7), indicating the deposition of ferric 
TABLE 3

Chemical Composition of Sediments in Averages and Their Standard Deviations

\begin{tabular}{|c|c|c|c|c|c|c|c|c|c|c|c|c|}
\hline & Site 434 & Site 435 & Site 436 & Site 438 & Site 439 & Site 440 & Site 441 & $\begin{array}{l}\text { Sikoku Basin } \\
\text { and Nankai } \\
\text { Trough }{ }^{3}\end{array}$ & Japan Trench ${ }^{b}$ & $\begin{array}{l}\text { Off Tsugaru } \\
\text { Japan Sea }\end{array}$ & $\begin{array}{l}\text { Central Japan } \\
\text { Sea }^{d}\end{array}$ & $\begin{array}{l}\text { Pacific Pelagic } \\
\text { Sediments }\end{array}$ \\
\hline $\begin{array}{l}\text { Numbers } \\
\text { of Sample }\end{array}$ & 54 & 24 & 66 & 52 & 12 & 39 & 10 & 29 & 82 & 87 & 69 & 35 \\
\hline $\mathrm{SiO}_{2}$ & $73.38 \div 4.22$ & $74.79 \div 6.05$ & $68.69 \pm 2.70$ & $75.65 \pm 4.63$ & $71.97 \pm 2.59$ & $74.91 \div 4.67$ & $74.13=3.25$ & $66.69 \pm 2.16$ & $68.84 \pm 2.78$ & $66.58 \pm 2.02$ & $65.80 \pm 3.84$ & 49.84 \\
\hline $\mathrm{TiO}_{2}^{-}$ & $0.51=0.074$ & $0.50 * 0.13$ & $0.58 \pm 0.096$ & $0.51 \pm 0.085$ & $0.59 \div 0.082$ & $0.54 \pm 0.11$ & $0.55 \pm 0.076$ & $0.72 \div 0.06$ & $0.62 \pm 0.068$ & $0.67 \pm 0.052$ & $0.73 \pm 0.11$ & 1.22 \\
\hline $\mathrm{Al}_{2} \mathrm{O}_{3}$ & $12.11=1.54$ & $11.27 \pm 2.77$ & $14.95=1.76$ & $11.50=2.18$ & $14.54=1.33$ & $12.09 \div 2.23$ & $12.84 \pm 1.65$ & $16.50=0.54$ & $13.60 \div 1.47$ & $16.12 \pm 0.63$ & $16.54 \pm 1.96$ & 17.38 \\
\hline $\mathrm{Fe}_{2} \mathrm{O}_{3}$ & $4.17 \pm 3.41$ & $2.96 \pm 1.81$ & $4.07 \div 1.24$ & $2.85 \div 0.82$ & $2.76 \div 0.84$ & $2.81=0.48$ & $2.88 \pm 0.41$ & & $2.41=1.06$ & $3.83 \neq 0.39$ & $1.90 \pm 0.59$ & \\
\hline FeO & $1.67 \pm 0.25$ & $1.61 \pm 0.42$ & $1.35 \pm 0.52$ & $1.74 \div 0.33$ & $1.95 \div 0.48$ & $1.76 \pm 0.41$ & $1.92 \pm 0.39$ & & $2.93 \pm 0.63$ & $1.81 \pm 0.29$ & $1.90=0.59$ & \\
\hline $\mathrm{MnO}$ & $0.12 \pm 0.10$ & $0.056 \pm 0.029$ & $0.26 \pm 0.51$ & $0.046=0.017$ & $0.045 \pm 0.022$ & $0.064 \div 0.050$ & $0.085 \pm 0.039$ & $0.087 \div 0.053$ & $0.067 \div 0.022$ & $0.069 \pm 0.042$ & $0.48 \pm 1.95$ & 1.98 \\
\hline $\mathrm{MgO}$ & $2.03 \div 0.44$ & $2.00 \pm 0.50$ & $2.18 \pm 0.68$ & $1.96 \div 0.43$ & $1.97 \pm 0.35$ & $2.14=0.54$ & $2.08 \pm 0.33$ & $2.29 \pm 0.33$ & $3.11=0.30$ & $3.31 \pm 0.42$ & $2.91 \pm 0.72$ & 3.48 \\
\hline $\mathrm{CaO}$ & $1.15=0.65$ & $1.85=0.61$ & $1.55 \pm 0.51$ & $1.60=0.95$ & $1.51=0.63$ & $1.38=0.45$ & $2.08=0.33$ & $1.52 \pm 0.90$ & $2.38 \pm 1.19$ & $1.93 \div 0.90$ & $1.04=0.65$ & 4.06 \\
\hline $\mathrm{Na}_{2} \mathrm{O}$ & $2.72 \pm 0.54$ & $3.05 \div 0.49$ & $3.54 \pm 0.42$ & $2.12 \pm 0.48$ & $2.29 \pm 0.43$ & $2.18=0.45$ & $2.10=0.35$ & $3.28 \div 0.34$ & $3.77 \pm 0.49$ & $3.00 \pm 0.54$ & $2.16 \pm 0.75$ & 5.39 \\
\hline $\mathrm{K}_{2} \mathrm{O}$ & $2.05 \div 0.30$ & $1.84 \pm 0.47$ & $2.75 \pm 0.45$ & $1.90 \pm 0.34$ & $2.28 \pm 0.21$ & $2.03 \div 0.37$ & $2.19=0.38$ & $3.08 \pm 0.38$ & $2.12=0.30$ & $2.49 \pm 0.28$ & $3.47 \pm 0.47$ & 3.01 \\
\hline $\mathrm{P}_{2} \mathrm{O}_{5}$ & $0.088 \div 0.028$ & $0.090=0.022$ & $0.10=0.056$ & $0.12 \pm 0.19$ & $0.092 \pm 0.024$ & $0.10 \pm 0.070$ & $0.087 \div 0.022$ & $0.14 \div 0.012$ & $0.15 \div 0.13$ & $0.17 \pm 0.15$ & $0.16=0.075$ & \\
\hline $\mathrm{Fe}_{2} \mathrm{O}_{3} / \mathrm{FeO}$ & $2.48 \div 0.11$ & $1.81=0.081$ & $6.63 \div 2.72$ & $1.66 \pm 0.077$ & $1.50=0.39$ & $1.65 \pm 0.083$ & $1.57 \pm 5.01$ & & $0.89 \pm 0.031$ & $2.16 \pm 0.023$ & $3.65=5.34$ & \\
\hline $\begin{array}{l}\text { Total le } \\
\text { as } \mathrm{Fe}_{2} \mathrm{O}_{3}\end{array}$ & $6.03=0.26$ & $4.74 \div 0.21$ & $5.57 \pm 0.33$ & $4.79 \pm 0.077$ & $4.39 \pm 0.21$ & $4.76 \pm 0.18$ & $5.01 \pm 0.45$ & $6.01 \pm 0.01$ & $5.67 \neq 0.024$ & $5.85 \pm 0.023$ & $6.94 \pm 1.54$ & 9.29 \\
\hline
\end{tabular}

Sugisaki (1978).
b Sugisaki and Honza (in press).

${ }_{\mathrm{d}}^{\text {Sugisaki (in press a). }}$.

${ }^{\mathrm{d}}$ Sugisaki (in press b).
Goldbelg and Arrhenius (1958). 


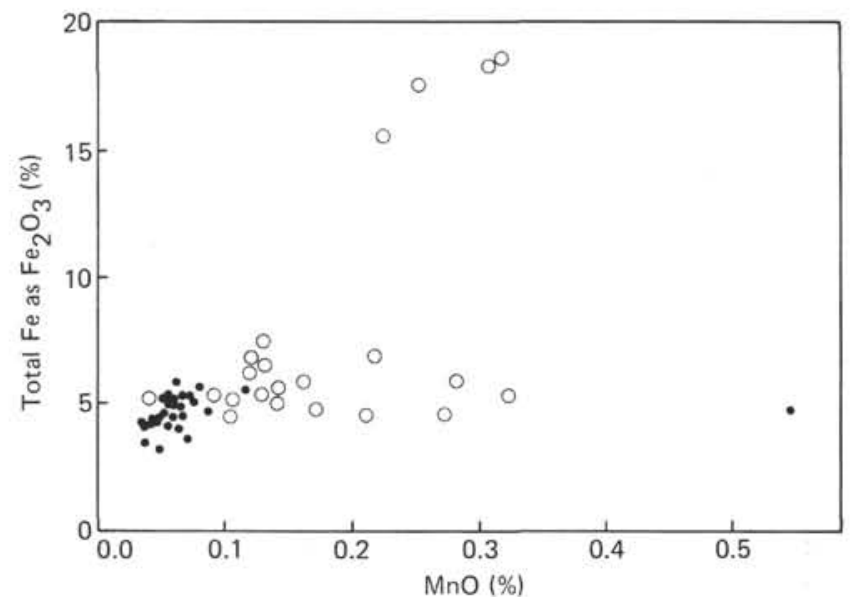

Figure 1. The relation between $\mathrm{MnO}$ and total $\mathrm{Fe}$ as $\mathrm{Fe}_{2} \mathrm{O}_{3}$ at Site 434. (Open circles $=$ samples containing $\mathrm{CaCO}_{3}$ and closed circles $=$ samples without $\left.\mathrm{CaCO}_{3}\right)$.

TABLE 4

Correlation Coefficients between $\mathrm{SiO}_{2}$ and Other Oxides

\begin{tabular}{|c|c|c|c|c|c|c|c|}
\hline & Site 434 & Site 435 & Site 436 & Site 438 & Site 439 & Site 440 & Site 441 \\
\hline $\mathrm{SiO}_{2}$ & 1.000 & 1.000 & 1.000 & 1.000 & 1.000 & 1.000 & 1.000 \\
\hline $\mathrm{TiO}_{2}^{2}$ & -0.323 & -0.929 & -0.868 & -0.864 & -0.877 & -0.933 & -0.923 \\
\hline $\mathrm{Al}_{2} \mathrm{O}_{3}$ & -0.400 & -0.952 & -0.885 & -0.946 & -0.839 & -0.992 & -0.922 \\
\hline $\mathrm{Fe}_{2} \mathrm{O}_{3}$ & -0.739 & -0.695 & -0.823 & -0.787 & -0.484 & -0.824 & -0.416 \\
\hline $\mathrm{FeO}$ & -0.513 & -0.947 & -0.271 & -0.763 & 0.087 & -0.888 & -0.538 \\
\hline $\mathrm{MnO}$ & -0.332 & -0.768 & -0.650 & -0.722 & 0.081 & -0.307 & -0.604 \\
\hline $\mathrm{MgO}$ & -0.680 & -0.900 & 0.082 & -0.744 & -0.492 & -0.920 & -0.701 \\
\hline $\mathrm{CaO}$ & -0.260 & -0.629 & 0.158 & -0.710 & -0.677 & -0.524 & -0.613 \\
\hline $\mathrm{Na}_{2} \mathrm{O}$ & -0.579 & -0.802 & 0.457 & -0.701 & -0.771 & -0.884 & -0.726 \\
\hline $\mathrm{K}_{2} \mathrm{O}$ & -0.346 & -0.783 & -0.363 & -0.756 & -0.456 & -0.910 & -0.538 \\
\hline $\mathrm{P}_{2} \mathrm{O}_{5}$ & -0.507 & -0.769 & -0.697 & 0.045 & -0.025 & -0.090 & -0.810 \\
\hline $\begin{array}{l}\text { Total } \mathrm{Fe} \\
\text { as } \mathrm{Fe}_{2} \mathrm{O}_{3}\end{array}$ & -0.765 & -0.813 & -0.878 & -0.887 & -0.488 & -0.927 & -0.886 \\
\hline
\end{tabular}

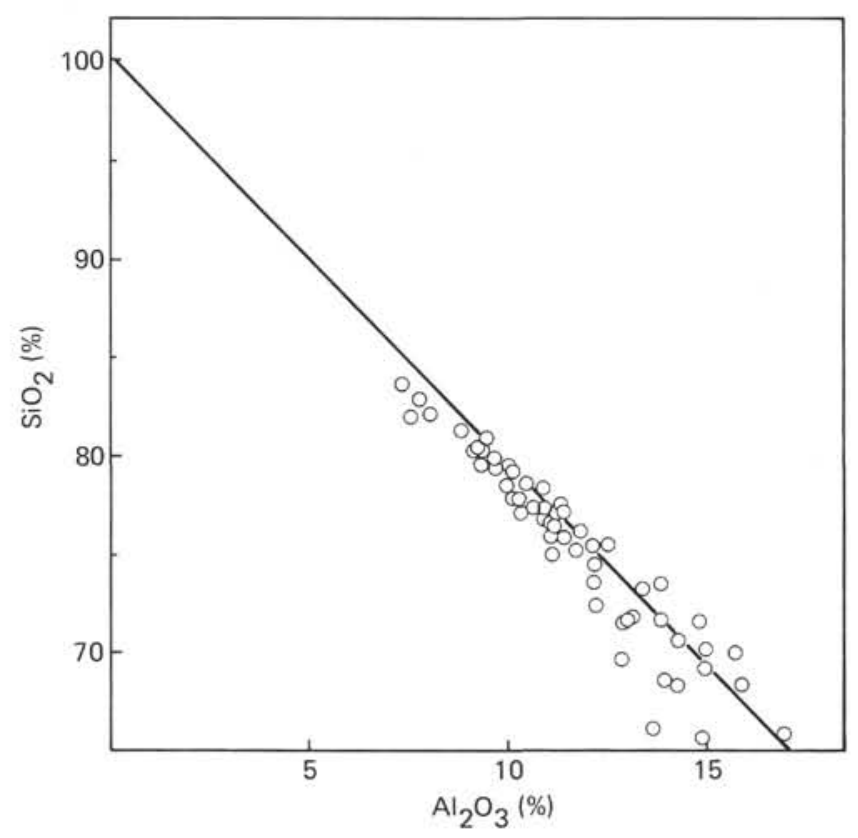

Figure 2. $\mathrm{SiO}_{2}-\mathrm{Al}_{2} \mathrm{O}_{3}$ relation at Site 438 (cf. Tables 5 and 6).
TABLE 5

$\mathrm{SiO}_{2}-\mathrm{Al}_{2} \mathrm{O}_{3}$ Relation by Least Squares Method

\begin{tabular}{ll}
\hline Site 434 & $\mathrm{SiO}_{2}=100.2-2.11 \mathrm{Al}_{2} \mathrm{O}_{3}{ }^{\mathrm{a}}$ \\
Site 435 & $\mathrm{SiO}_{2}=99.40-2.18 \mathrm{Al}_{2} \mathrm{O}_{3}$ \\
Site 436 & $\mathrm{SiO}_{2}=91.62-1.53 \mathrm{Al}_{2} \mathrm{O}_{3}$ \\
Site 438 & $\mathrm{SiO}_{2}=100.1-2.12 \mathrm{Al}_{2} \mathrm{O}_{3}$ \\
Site 439 & $\mathrm{SiO}_{2}=100.3-1.94 \mathrm{Al}_{2} \mathrm{O}_{3}$ \\
Site 440 & $\mathrm{SiO}_{2}=99.14-2.00 \mathrm{Al}_{2} \mathrm{O}_{3}$ \\
Site 441 & $\mathrm{SiO}_{2}=99.42-1.97 \mathrm{Al}_{2} \mathrm{O}_{3}$ \\
\hline
\end{tabular}

${ }^{\mathrm{a}}$ This formula was calculated excluding 7 samples of high content of iron (Figure 1). The formula for all samples at Site 434 is $\mathrm{SiO}_{2}=106.6-2.74 \mathrm{Al}_{2} \mathrm{O}_{3}$.

TABLE 6

Weight Per Cent of $\mathrm{SiO}_{2}$ Extrapolated from Regression Equations for Each Oxide, Site 438

\begin{tabular}{lc}
\hline $\mathrm{TiO}_{2}$ & 103.6 \\
$\mathrm{Al}_{2} \mathrm{O}_{3}$ & 100.1 \\
$\mathrm{Fe}_{2} \mathrm{O}_{3}$ & 91.75 \\
$\mathrm{FeO}$ & 99.88 \\
$\mathrm{MnO}$ & 88.18 \\
$\mathrm{MgO}$ & 96.81 \\
$\mathrm{CaO}$ & 83.40 \\
$\mathrm{Na}_{2} \mathrm{O}$ & 96.27 \\
$\mathrm{~K}_{2} \mathrm{O}$ & 101.4 \\
$\mathrm{P}_{2} \mathrm{O}_{5}$ & 78.56 \\
$\mathrm{Total} \mathrm{Fe}$ & 96.83 \\
as $\mathrm{Fe}$ & $\mathrm{O}_{3}$ \\
\hline
\end{tabular}

iron in each site. The increase of $\mathrm{Fe}_{2} \mathrm{O}_{3}$ may be due to the inorganic precipitation from the sea water under oxidizing conditions. On the other hand, $\mathrm{FeO}$ content does not fluctuate as much as $\mathrm{Fe}_{2} \mathrm{O}_{3}$. This implies that $\mathrm{FeO}$ from original sources was not oxidized but remained unchanged in the hemipelagic zone, although the iron in deep sea sediments is generally considered to be mostly oxidized into $\mathrm{Fe}_{2} \mathrm{O}_{3}$. The present interpretation supports the inference in the preceding section that $\mathrm{Fe}_{2} \mathrm{O}_{3}$ coprecipitated with silica whereas $\mathrm{FeO}$ did not.

As I stated earlier, although manganese also appears to precipitate with silica, the $\mathrm{MnO}$ content at each site is generally low. This may be attributed to the following mechanisms. (1) $\mathrm{MnO}$ does not precipitate as much as $\mathrm{Fe}_{2} \mathrm{O}_{3}$ in a hemipelagic environment. (2) Precipitated $\mathrm{MnO}$ is confined to sediments which accumulated rapidly. (3) $\mathrm{MnO}$ dissolves under a reducing condition created by organic materials derived from the lands. The redox potential at $p \mathrm{H} 8$ for oxidation reaction from $\mathrm{Fe}^{++}$to $\mathrm{Fe}^{+++}$is -0.2 volts, whereas that from $\mathrm{Mn}^{++}$ to $\mathrm{Mn}^{++++}$is +0.5 volts (Pourbaix, 1963). Manganese is therefore easily reduced and barely precipitated com- 

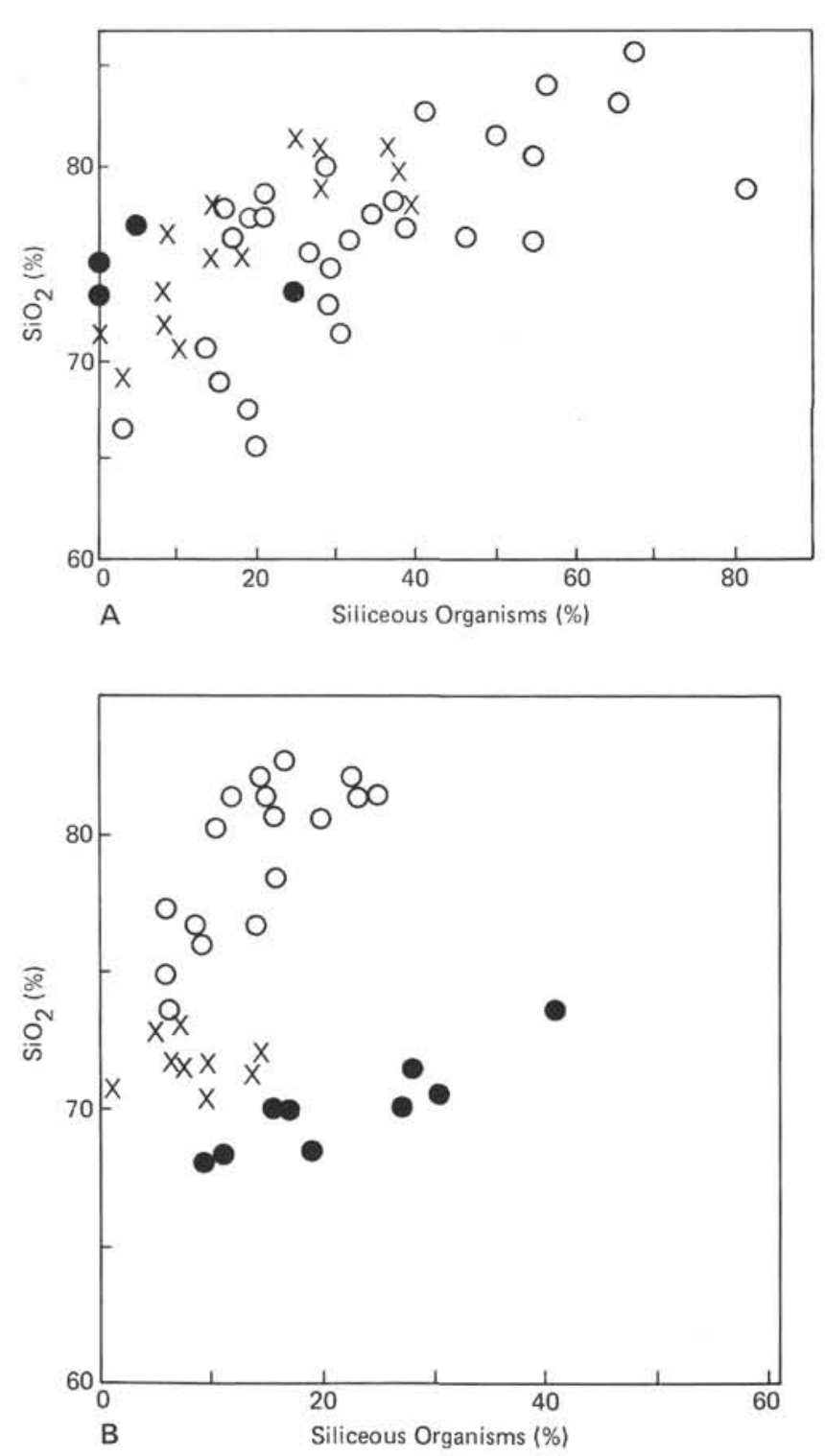

Figure 3. A. The relationship between $\mathrm{SiO}_{2}$ and siliceous organisms content (diatom, radiolarian, and sponge) at Site 438. (Open circles $=$ samples shallower than 600 meters. Closed circles $=$ samples with more than 60 per cent volcanic glass. $\times=$ samples deeper than 600 meters. Regression line for shallower samples excluding the closed circles is $\mathrm{SiO}_{2}=66.52+0.284$ [organism content], with a correlation coefficient of 0.644. For deeper samples it is $\mathrm{SiO}_{2}=69.95+0.330$ [organism content], with a correlation coefficient of 0.789.) B. The relationship between $\mathrm{SiO}_{2}$ and siliceous organisms at Site 440. (Closed circles $=$ samples shallower than 200 meters. Regression line is $\mathrm{SiO}_{2}=66.33$ +0.166 [organism content], with a correlation coefficient of 0.891. Open circles $=$ samples deeper than 350 meters. Regression line is $\mathrm{SiO}_{2}=72.48+0.451$ [organism content], with a correlation coefficient of $0.503 . \times=$ samples between 200 and 350 meters in depth.)

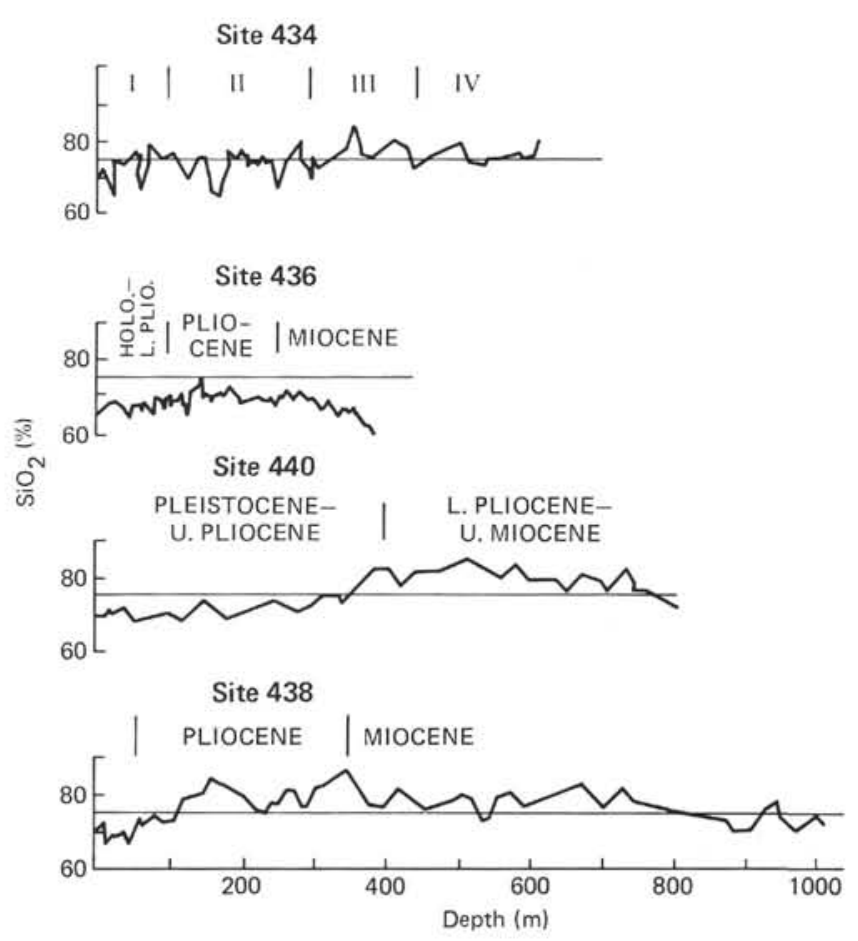

Figure 4. $\mathrm{SiO}_{2}$ variation with depth. For Site 434, the geological age is obscure because of repetition of the biostratigraphic horizon; sections deeper than 300 meters are regarded as late Miocene (Roman numerals for Site 434 represent lithologic units: $I=$ diatomaceous ooze, $I I=$ spicular diatomaceous mudstone and diatomaceous-spicular mudstone, $I I I=$ vitric diatomaceous mudstone and diatomaceous vitric mudstone, $I V=$ tuffite.)

pared with iron. $\mathrm{CH}_{4}$ and $\mathrm{H}_{2} \mathrm{~S}$ at Site 434 , on the west slope of the trench, show the reducing environment. As I discuss later, Site 436 , on the east side of the trench, displays higher $\mathrm{MnO}$ content and a higher $\mathrm{Fe}_{2} \mathrm{O}_{3} / \mathrm{FeO}$ ratio, suggesting an oxidizing environment.

\section{ORIGIN OF THE WEST SLOPE SEDIMENTS OF THE JAPAN TRENCH}

Figure 6 illustrates the relationship among $\mathrm{SiO}_{2}$, $\mathrm{TiO}_{2}$, and $\mathrm{Al}_{2} \mathrm{O}_{3}$. These elements tend to remain in the resistates and hydrolyzates during sedimentation processes (Rankama and Sahama, 1950). Because the mutual abundance of these conservative elements probably remains stationary during weathering, the diagram is expected to be helpful in examining sediment sources. Because most samples are contaminated by biogenic silica, only those with $\mathrm{Al}_{2} \mathrm{O}_{3}$ content higher than 14 per cent were plotted. The points are located along a line connecting averaged Japanese granites and averaged mudstones from the Chichibu geosynclinal areas. The distribution on the diagram exhibits a striking contrast with that of argillaceous sediments from the Nankai Trough and the Shikoku Basin (Sugisaki, 1978) and the Japan Sea (Sugisaki, 1979, in press a). The points from these regions are located between averaged Japanese granites and averaged Quaternary volcanics. The Kitakami high- 


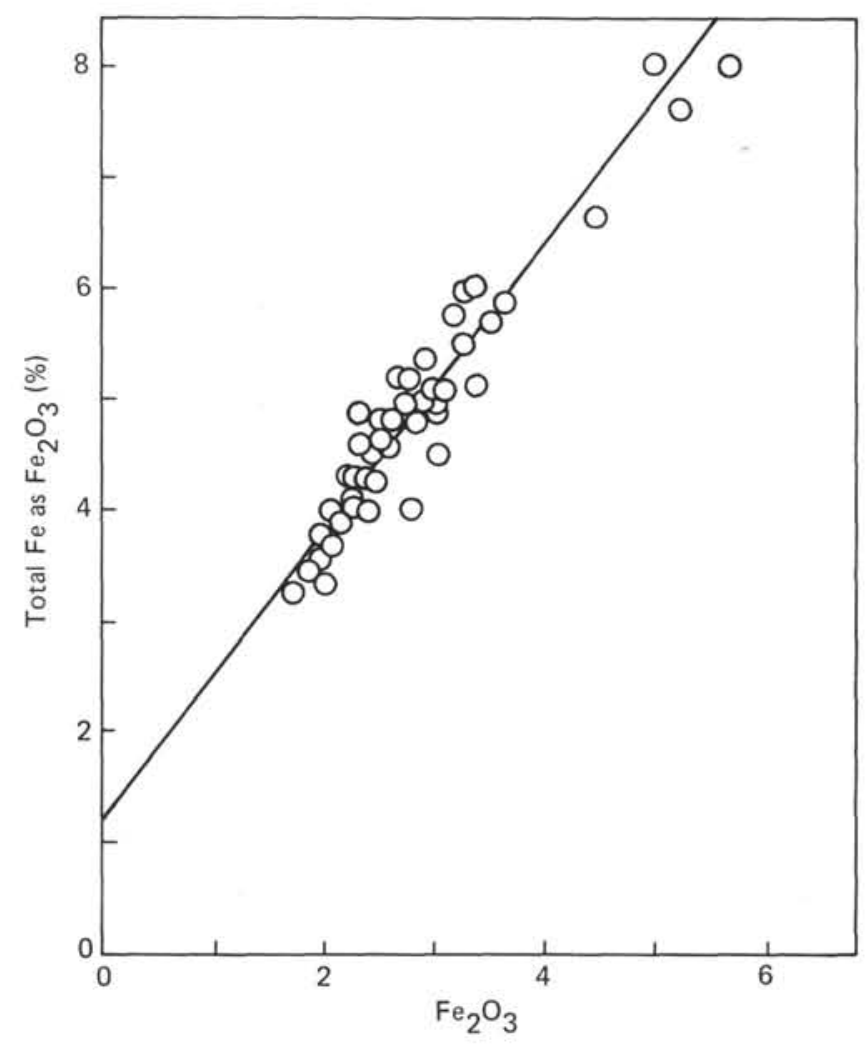

Figure 5. The relationship between $\mathrm{Fe}_{2} \mathrm{O}_{3}$ and total $\mathrm{Fe}$ as $\mathrm{Fe}_{2} \mathrm{O}_{3}$ at Site 438 .

TABLE 7

$\mathrm{Fe}_{2} \mathrm{O}_{3}$ - Total $\mathrm{Fe}$ as $\mathrm{Fe}_{2} \mathrm{O}_{3}$ Relation by Least Squares Method

\begin{tabular}{ll}
\hline Site 434 & Total $\mathrm{Fe}=1.021 \mathrm{Fe}_{2} \mathrm{O}_{3}+1.756$ \\
Site 435 & Total $\mathrm{Fe}=1.155 \mathrm{Fe}_{2} \mathrm{O}_{3}+1.329$ \\
Site 438 & Total $\mathrm{Fe}=1.277 \mathrm{Fe}_{2} \mathrm{O}_{3}+1.150$ \\
Site 439 & Total $\mathrm{Fe}=0.880 \mathrm{Fe}_{2} \mathrm{O}_{3}+2.50$ \\
Site 440 & Total $\mathrm{Fe}=1.80 \mathrm{Fe}_{2} \mathrm{O}_{3}+0.301$ \\
Site 441 & Total $\mathrm{Fe}=1.097 \mathrm{Fe}_{2} \mathrm{O}_{3}+1.852$ \\
\hline
\end{tabular}

lands, which are the hinterland of the area of Legs 56 and 57, are composed mainly of granites and Chichibu geosynclinal sediments. This suggests that the sediments derived mainly from the land area to the west, comprising the Kitakami and Abukuma highlands, Hokkaido, and other land areas, and that the original materials, which were chemically homogeneous, suffered subsequent contamination by biogenic silica.

Many samples contain volcanic glass, as previously stated. Since the composition of the glass was not determined, only the samples with a volcanic glass content greater than 60 per cent and at a location nearest to the samples selected for chemical analyses were plotted on the diagram. These points deviate from those of the closed circle samples and move toward the $\mathrm{SiO}_{2}$ apex of the diagram. The high content of $\mathrm{SiO}_{2}$ in some samples may be due partly to high amounts of volcanic glass.

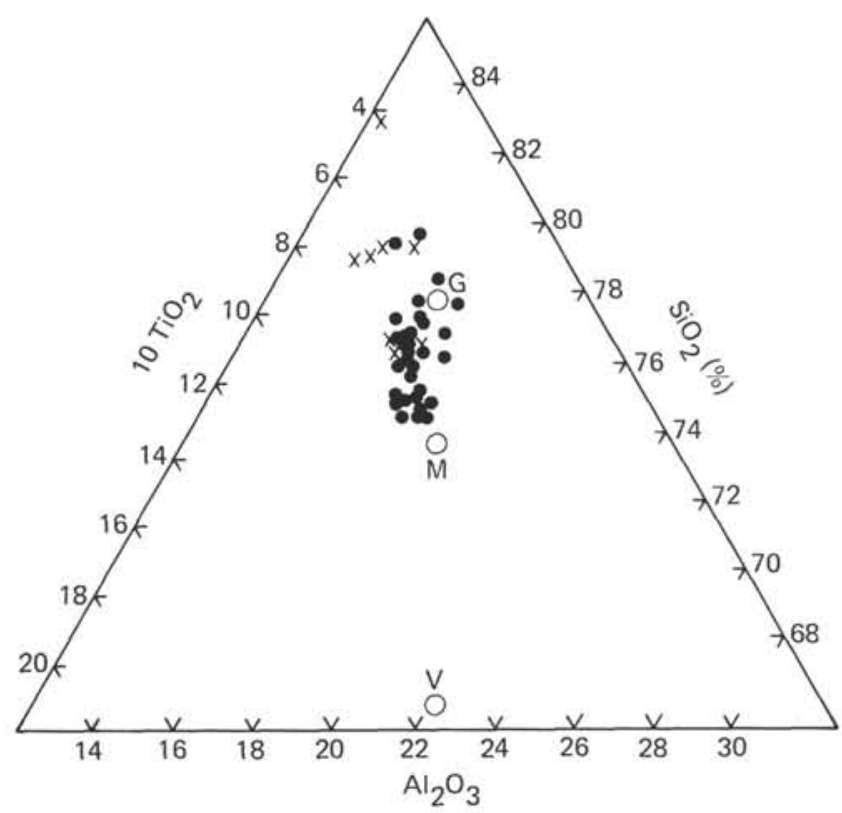

Figure 6. Relationship of $\mathrm{SiO}_{2}-\mathrm{TiO}_{2}-\mathrm{Al}_{2} \mathrm{O}_{3}$ on the west slope of the Japan Trench. (Closed circles $=$ samples with more than 14 per cent $\mathrm{Al}_{2} \mathrm{O}_{3} . \times=$ samples with more than 60 per cent volcanic glass. Open circles $=$ averaged Japanese rocks. $G=$ granite [calculated from 440 analyses by the Geological Survey of Japan, 1960], $M=$ mudstones from the Chichibu geosynclinal area [300 analyses, Katada et al., 1977], $V=$ quaternary volcanic rocks [769 analyses, Sugisaki, 1970].)

\section{CHEMICAL TREND AT SITE 436}

Core recovered from Site 436, on the east slope of the Japan Trench, displays a striking chemical variation from cores from the west slope. As sample depth increases, $\mathrm{SiO}_{2}$ content decreases, whereas the $\mathrm{MnO}$ content and $\mathrm{Fe}_{2} \mathrm{O}_{3} / \mathrm{FeO}$ ratios increase noticeably. The $\mathrm{MnO}$ content of the three deepest samples is almost 2.5 per cent, or about 50 times as high as the upper part of the core. The deep samples characterized by high $\mathrm{MnO}$ and $\mathrm{Fe}_{2} \mathrm{O}_{3}$ content and low $\mathrm{SiO}_{2}$ content are closely similar if not identical to the pelagic sediments from the Pacific Ocean listed in Table 3. By contrast, the upper part of the core is chemically similar to the west slope sediments, which derived from the Japanese Islands and were contaminated by the biogenic silica.

The $\mathrm{SiO}_{2}-\mathrm{TiO}_{2}-\mathrm{Al}_{2} \mathrm{O}_{3}$ diagram in Figure 7 illustrates that samples from the upper part of the section (Holocene to late Miocene) derive mainly from the same area as for the west slope sediments (cf. Figure 6), whereas samples from the lower section (Miocene and older) deviate downward, indicating different sources for deeper and shallower sediments.

The vertical tendency of the core at Site 436 can be explained by the movement of the ocean plate. The $\mathrm{MnO}$ content was plotted against the absolute age of each sample, which was tentatively assigned by the interpolation of the paleontological age of the core (Figure 8). $\mathrm{MnO}$ 


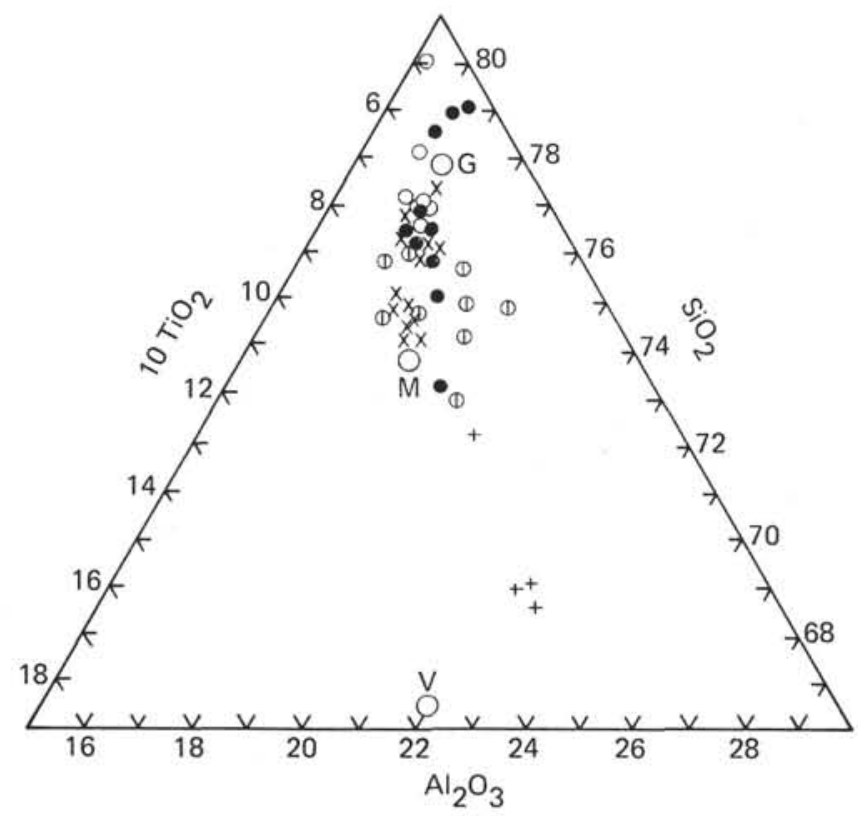

Figure 7. Relationship of $\mathrm{SiO}_{2}-\mathrm{TiO}_{2}-\mathrm{Al}_{2} \mathrm{O}_{3}$ at Site 436. $(\times=$ Holocene to late Pliocene samples. Open circles $=$ Pliocene. Closed circles = late Miocene. Bisected circles $=$ Miocene. . Crosses $=$ oldest samples in the core.)

content increases exponentially with time. When the oceanic plate was in the Central Pacific Ocean, $\mathrm{MnO}$ and $\mathrm{Fe}_{2} \mathrm{O}_{3}$ were deposited under an oxidizing environment. As the ocean plate moved westward and approached the Japanese Islands, the tremendous volume of hemipelagic sediments, containing much organic material derived from the islands, gradually deposited over the original pelagic sediments. The continuous movement at a uniform rate of the plate toward the Japanese Islands gave rise to the chemical trend exemplified by the progressive change of $\mathrm{MnO}$.

\section{CONCLUSIONS}

It should again be emphasized that the sediments on the west slope of the Japan Trench were derived chiefly from the Japanese Islands and were accompanied by the precipitation of biogenic silica and ferric iron. Deeper sediment overlying oceanic crust (Site 436) consists of typical pelagic material which was conveyed by the plate movement from the East Pacific Ocean. From the viewpoint of plate convergence, the upper part of the subducted oceanic lithosphere is generally assumed to have accreted to the inner trench slope via offscraping (e.g., Karig and Sharman, 1975). But the picture is by no means as simple as it was once thought to be (e.g., Scholl et al., 1977). If the offscraping of the oceanic plate is the dominant process at a convergent margin, a significant amount of deep oceanic pelagic sediment scraped off the plate should be contained in the accreting wedge. Yet the present study presents conflicting evidence. No pelagic sediments were geochemically detected in the trench inner slope deposits, though the possibility re-

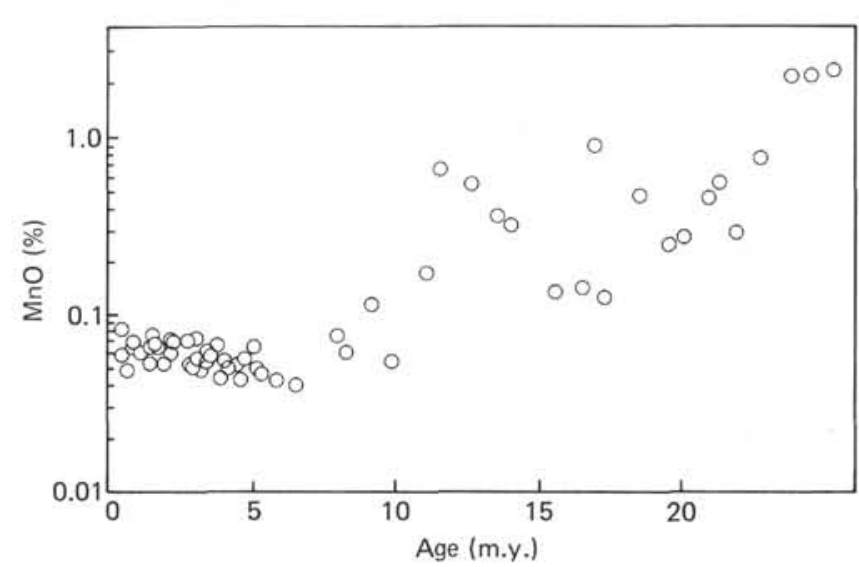

Figure 8. $\mathrm{MnO}$ variation with age in the sediments at Site 436. Age was assigned by the interpolation of the paleontological age.

mains that pelagic sediments occur at greater depths, under the trench inner slope, and that the present drilling cores did not reach those depths. When we remember that typical pelagic sediments occur at the outer slope of the trench, as observed at Site 436, it seems likely that much of the pelagic sediment is subducted without being offscraped as suggested by volume calculations (e.g., von Huene, Nasu, et al, Site 441 chapter).

\section{ACKNOWLEDGMENTS}

I am grateful to Dr. H. Okada, Shizuoka University, and Dr. T. Moritani, Geological Survey of Japan, for their critical reading of the manuscript. I also thank Dr. S. Mizutani, Nagoya University, for his valuable comments.

\section{REFERENCES}

Geological Survey of Japan, 1960. Geology and mineral resources of Japan.

Goldberg, E. D., and Arrhenius, G. O. S., 1958. Chemistry of Pacific pelagic sediments. Geochim. Cosmochim. Acta, 13, 153-213.

Karig, D. E., and Sharman, G. E., 1975. Subduction and accretion in trenches. Geol. Soc. Am. Bull., 86, 377-389.

Kastner, M., Keene, J. B., and Gieskes, J. M., 1977. Diagenesis of siliceous ooze. 1. Chemical controls on the test of opalCT transformation-an experimental study. Geochim. Cosmochim. Acta., 41, 1041-1059.

Katada, M., Tanji, K., Ono, C., and Teraoka, Y., 1977. Average chemical compositions of rocks and their graphic representation. 6. Tertiary mudstone. Bull. Geol. Surv. Jpn., 28, 327-342.

Pourbaix, M., 1963. Atlas d'Équilibres Électrochimiques à $25^{\circ} \mathrm{C}$ : Paris (Gauthier-Villars).

Rankama, K., and Sahama, H., 1950. Geochemistry: Chicago (University of Chicago Press).

Scholl, D. W., Marlow, M. S., and Cooper, A. K., 1977. Sediment subduction and offscraping at Pacific margins. In Talwani, M., and Pitman, W. C. (Eds.), Island Arcs, Deep Sea Trenches and Back Arc Basins: Washington (American Geophysical Union), pp. 199-210.

Shapiro, L., and Brannock, W. W., 1955a. Rapid determination of water in silicate rocks. Anal. Chem., 27, 560-562. 1955b. Rapid determination of carbon dioxide in silicate rocks. Anal. Chem., 27, 1796-1797. 
Sugisaki, R., 1976. Chemical characteristics of volcanic rocks: Relation to plate movements. Lithos, 9, 17-30.

, 1978. Chemical composition of argillaceous sediments on the Pacific margins of southwest Japan. In Inoue, E. (Ed.), Cruise Report No. 9: Kawasaki (Geological Survey of Japan), pp. 65-73.

1979. Chemical composition of argillaceous sediments around the Yamato Banak in the Japan Sea. In Honza, E. (Ed.), Cruise Report No. 9: Kawasaki (Geological Survey of Japan), pp. 75-88. , in press a. Chemical composition of argillaceous sediments around the Nishitsugaru Basin in the Japan Sea. In
Inoue, E. (Ed.), Cruise Report: Kawasaki (Geological Survey of Japan).

, in press b. A modified method of analysis of bulk chemical composition of argillaceous rocks and data display - with special reference to marine sediments. J. Geol. Soc. Jpn.

Sugisaki, R., and Honza, E., in press. Chemical composition of argillaceous sediments in the Pacific margin of northeast Japan. Bull. Geol. Surv. Jpn.

Sugisaki, R., Shimomura, T., and Ando, K., 1977. An automatic X-ray fluorescence method for the analysis of silicate rocks. J. Geol. Soc. Jpn., 83, 710-722. 